\title{
Closing in on compressed gluino-neutralino spectra at the LHC
}

\author{
Guillaume Chalons and Dipan Sengupta \\ Laboratoire Physique Subatomique et Cosmologie, Université Grenoble-Alpes, \\ CNRS/IN2P3, Grenoble INP, 53 rue des Martyrs, F-38026 Grenoble, France \\ E-mail: chalons@lpsc.in2p3.fr, dipan@lpsc.in2p3.fr
}

ABStract: A huge swath of parameter space in the context of the Minimal Supersymmetric Standard Model (MSSM) has been ruled out after Run I of the LHC. Various exclusion contours in the $m_{\tilde{g}}-m_{\tilde{\chi}_{1}^{0}}$ plane were derived by the experimental collaborations, all based on three-body gluino decay topologies. These limits are however extremely model dependent and do not always reflect the level of exclusion. If the gluino-neutralino spectrum is compressed, then the current mass limits can be drastically reduced. In such situations, the radiative decay of the gluino $\tilde{g} \rightarrow g \tilde{\chi}_{1}^{0}$ can be dominant and used as a sensitive probe of small mass splittings. We examine the sensitivity of constraints of some Run I experimental searches on this decay after recasting them within the MadAnalysis5 framework. The recasted searches are now part of the MadAnalysis5 Public Analysis Database. We also design a dedicated search strategy and investigate its prospects to uncover this decay mode of the gluino at Run II of the LHC. We emphasize that a multijet search strategy may be more sensitive than a monojet one, even in the case of very small mass differences.

Keywords: Supersymmetry Phenomenology

ARXIV EPRINT: 1508.06735 


\section{Contents}

1 Introduction and motivation $\quad 1$

2 Phenomenology of the radiative gluino decay 4

3 Constraining the gluino loop decay from existing 8 TeV LHC analyses 10

4 Prospects at the 13 TeV LHC $\quad 15$

5 Conclusion $\quad 20$

$\begin{array}{ll}\text { A Cutflow for the ATLAS monojet analysis } & 21\end{array}$

B Cutflow for the ATLAS multi-jet analysis 21

\section{Introduction and motivation}

During Run I of the LHC, in addition to the quest for the Higgs boson, an extensive search programme for New Physics (NP) phenomena has been carried out. However, no sign of physics beyond the Standard Model (BSM) has been unravelled so far [1-4]. As a result the parameter space for weak scale NP, including models of weak scale Supersymmetry (SUSY), has shrunk considerably.

The simplest incarnations of weak scale SUSY, the MSSM and its variants, depending on the SUSY breaking scheme, are facing a wealth of experimental data [1-4], including the Higgs boson mass measurement $m_{H} \simeq 125 \mathrm{GeV}$ [5]. Since no NP signal was revealed, the only appropriate physical interpretation of these searches was to set limits on cross sections for the production of SUSY particles and then derive bounds on their masses. Interpreting the NP experimental searches is a non-trivial task and almost impossible to perform in a model-independent way. The early searches for SUSY phenomena have focussed on the constrained MSSM (CMSSM)/minimal supergravity (mSUGRA) and probed mainly the gluino $(\tilde{g})$ and the squarks $(\tilde{q})$ of the first two generations $[6,7]$. The current lower limits on the gluino and the first two generations squark masses in the framework of the CMSSM stand at $m_{\tilde{g}} \gtrsim 1.7 \mathrm{TeV}$ for almost degenerate gluino and squarks and $m_{\tilde{q}}>1.4 \mathrm{TeV}$ for very high squark masses. However, the universal boundary conditions imposed at the Grand Unified Theory (GUT) scale in such models impose particular relations between particle masses, decay branching ratios, etc... which severely restrict the way a SUSY signal could show up. To relax some of these assumptions, the Simplified Model Spectra (SMS) [812] approach has been adopted systematically by the ATLAS and CMS collaborations to interpret the results. Instead of considering the full SUSY spectrum, a SMS search only 
targets one or two relevant decay modes (and the particles involved in) with $100 \%$ branching ratio (BR). In turn, a handful of parameters are to be considered for interpreting the analysis: the production cross section of the mother particle, its mass, and the ones of the daughters. Nevertheless, such SMS approaches may not cover all patterns in which a NP signal could be unveiled, especially if one consider the plethora of existing supersymmetric models. More precisely, even in R-parity conserving scenarios, if the lightest SUSY particle (LSP) is massive and degenerate with the squarks and/or gluinos, the so-called "compressed" SUSY scenarios, these limits can be seriously weakened [13-19].

As far as the official experimental analyses are concerned, published gluinos mass limits in the SMS approach were obtained mainly from its three-body decays $\tilde{g} \rightarrow q \bar{q} \tilde{\chi}_{1}^{0}$, where the final states quark can be of any flavour [6, 20-24] or $\tilde{g} \rightarrow q q^{\prime} \tilde{\chi}_{1}^{ \pm}[6,20]$. Provided the gluino-neutralino spectrum is compressed, the limits derived from three-body decays involving heavy-flavours do not apply and only the ones with light-flavours in the final states can give a handle on this situation. However, as limits on the first and second generation of squarks are quite strong, the $\tilde{g} \rightarrow q \bar{q} \tilde{\chi}_{1}^{0}$ modes with light quarks may be heavily suppressed. Furthermore, as already stressed, a large part of the parameter space feature a compressed spectra to evade the stringent bounds from the LHC, and few SMS interpretations fully encompass this situation for the gluino. In compressed scenarios, initial state radiation (ISR) in the form of jets which recoil against the missing momentum of the LSP may be used to discover or place bounds on the model. This method has been used to set mass limits on squarks and gluinos [6, 13-19, 25-29] and third generation squarks [13, 28, 30-34].

In the case where the three body decays are kinematically forbidden or suppressed, there exists another decay pattern available for the gluinos to decay into, which has received very little attention so far, but could fill the small mass difference gap: that of the two-body radiative decay $\tilde{g} \rightarrow g \tilde{\chi}_{i}^{0}$.

In a CMSSM-inspired scenario, since the mass difference $\Delta M=m_{\tilde{g}}-m_{\tilde{\chi}_{1}^{0}}$ is fixed from conditions imposed at the GUT scale, when $m_{\tilde{g}}$ increases, so does $\Delta M$. In turn, the decay width of the gluino will be dominated by the three-body decays. Unlike GUT constrained SUSY-scenarios, general explorations of the MSSM may exhibit significant portions of parameter space where the radiative decay $\tilde{g} \rightarrow g \tilde{\chi}_{1}^{0}$ can be competitive with three-body decays [35-42]. Within these specific regions, significant branching fractions of the gluino radiative decay are characterised by a spectrum with heavy squarks. Thus, the direct two-body decays $\tilde{g} \rightarrow \tilde{q} q$ are forbidden and $\tilde{g} \rightarrow q \bar{q} \tilde{\chi}_{i}^{0}$ are suppressed by the heavy scalar quark mass, leaving the loop-induced decay as the only available mode to decay into. Therefore, for compressed gluino-neutralino scenarios, the gluino loop decay can be used as a sensitive probe and improve the current limits.

A spectrum with decoupled scalars and lighter gauginos and higgsinos is not unexpected in SUSY models like (mini) Split-SUSY/PeV scale SUSY [43-49], Spread SUSY scenarios [50] or pure gravity mediation [49, 51-53]. Moreover, a Higgs mass around $125 \mathrm{GeV}$ implies a non-negligible branching fraction of the gluino loop decay when $\tan \beta \simeq \mathcal{O}(1)$ [42]. It is worth mentioning that if the scalar superpartners are heavy enough, then the gluino lifetime $\tau_{\tilde{g}}$ can be sufficiently large to lead to displaced vertices or even hadronise into R-hadrons $[46,47,54,55]$. Nevertheless, if $\tilde{m} \lesssim \mathcal{O}\left(10^{4}\right) \mathrm{TeV}, \tau_{\tilde{g}}$ is too small such that the gluino decays promptly in the detector [42]. 
To obtain a compressed gluino-neutralino spectrum, given a SUSY-breaking scheme, one needs non-universalities in the gaugino sector at the GUT scale to obtain a small $\Delta M$. In the context of non-universal supergravity (NUSUGRA) models, the gluino can be in a significant part of the parameter space the next-to-lightest SUSY particle (GNLSP) and the neutralino the LSP [56-63]. A non-exhaustive list of other SUSY models accommodating the GNLSP scenario include left-right symmetric model with gravity mediated SUSY breaking [64-67], pure gravity mediation/mini-split SUSY [49, 53] and general gauge mediation [68].

Besides being a discovery mode for the gluino, its loop-decay has some other merits from the phenomenological perspective. Indeed, since squarks contribute to the radiative decay through loop diagrams, the two-body decays $\tilde{g} \rightarrow g \tilde{\chi}_{i}^{0}$ carry important information about the scalar mass scale $\tilde{m}$ [42], which would be otherwise inaccessible in scenarios with decoupled scalars. Moreover, in GNLSP models, the LSP can account for the relic density of dark matter through its coannihilation with the gluino. The efficiency of such processes crucially depends on the gluino-LSP mass difference $\Delta M$. Thus, measuring or constraining this splitting, only accessible at collider experiments, is essential to estimate the phenomenological viability of these models.

We should also note in passing, that in models with spontaneously broken global supersymmetry the radiative decay $\tilde{g} \rightarrow g \widetilde{G}$, where $\widetilde{G}$ is the gravitino, is also possible. It can be dominant when the original scale of supersymmetry breaking $\sqrt{F}$ is of the same order of the squark mass scale $\widetilde{m}[41,45,69-76]$, whatever the $m_{\tilde{g}}-m_{\widetilde{G}}$ mass difference. Depending on the value of $F$ the gluino can decay promptly, in flight inside the detector, or be long-lived. For very long-lived gluinos, ATLAS and CMS have excluded charged R-hadrons with a mass less than $1.3 \mathrm{TeV}[77,78]$, if they escape the detector, or less than $850 \mathrm{GeV}$ [54] if stopped in the detector. If metastable, the gluino is excluded up to $m_{\tilde{g}}=850 \mathrm{GeV}$ for decays to $q \bar{q} \tilde{\chi}_{1}^{0} / g \tilde{\chi}_{1}^{0}$, for a lifetime of $1 \mathrm{~ns}$ and $m_{\tilde{\chi}_{1}^{0}}=100 \mathrm{GeV}$ [79].

A work probing scenarios where the LSP and the gluino are almost degenerate was recently performed in [28]. However, the limits derived there solely considered the three-body decays of the gluino into light-flavour quarks $\tilde{g} \rightarrow q \bar{q} \tilde{\chi}_{1}^{0}$, but with light sbottoms and light stops in the spectrum. As we will review in the forthcoming sections, the radiative decay $\tilde{g} \rightarrow g \tilde{\chi}_{1}^{0}$ is favoured when light stops are present and should dominate instead. Therefore, investigating rather the gluino loop-induced decay seems better motivated from a phenomenological point of view when all scalars are heavy and $\Delta M$ small. The ATLAS collaboration has also recently provided, using only a hadronic search that we reimplemented for this work, some results on the radiative gluino decay in [6]. Yet, results have only been presented for a fixed gluino mass $m_{\tilde{g}}$ or fixed LSP mass $m_{\tilde{\chi}_{1}^{0}}$, but not in the full $m_{\tilde{g}}-m_{\tilde{\chi}_{1}^{0}}$ plane.

In this work, we aim at quantifying to which extent the radiative gluino decay can constrain, using Run I monojet and multijets analyses, the region where the mass splitting $\Delta M=m_{\tilde{g}}-m_{\tilde{\chi}_{1}^{0}}$ is small, assuming a SMS interpretation where $\operatorname{BR}\left(\tilde{g} \rightarrow g \tilde{\chi}_{1}^{0}\right)=100 \%$. We argue in the next section, after investigating the parameter space where the loop decay is important, why a somewhat lighter third generation squark spectrum is desirable to enhance this decay from the phenomenological MSSM (pMSSM) point of view. We use the MadAnalysis5 $[80,81]$ framework to derive exclusion contours in the $m_{\tilde{g}}-m_{\tilde{\chi}_{1}^{0}}$ plane, 


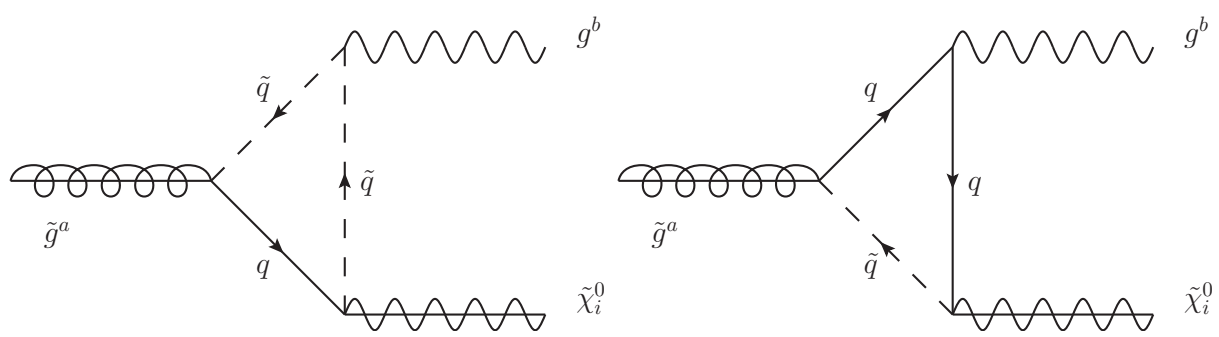

Figure 1. Diagrams relevant for the radiative gluino decay. Since the gluino is a Majorana fermion each diagram is accompanied with a similar one but with the internal arrows reversed.

deduced from a Simplified Model approach, after recasting ATLAS and CMS monojet and multijets analyses. We provide in our work a full quantitative exploration of this plane and the quoted ATLAS 95\% CL upper bound [6] for fixed $m_{\tilde{g}}$ and $m_{\tilde{\chi}_{1}^{0}}$ rather serves to us as a cross check on our results, which give us quite confidence in our procedure with which we derived our own mass limits. These recasted analyses are now available on the MadAnalysis5 Public Analysis Database (PAD) [82]. The last section is devoted to the determination of a search strategy at $13 \mathrm{TeV}$ to investigate the prospects for discovering the gluino through its radiative decay at Run II of the LHC and then we conclude.

\section{Phenomenology of the radiative gluino decay}

In this section we review the gluino decays in a heavy scalar scenario. When the squarks are heavier than the gluino, the latter can undergo three body decays into a pair of quarks and a neutralino or chargino through an off-shell intermediate squark, or decay radiatively into a gluon and a neutralino,

$$
\begin{aligned}
& \tilde{g} \rightarrow q+\bar{q}+\tilde{\chi}_{i}^{0} \\
& \tilde{g} \rightarrow q+\bar{q}^{\prime}+\tilde{\chi}_{j}^{ \pm} \\
& \tilde{g} \rightarrow g+\tilde{\chi}_{i}^{0}
\end{aligned}
$$

where $\tilde{\chi}_{i}^{0}(i=1 \ldots 4)$ and $\tilde{\chi}_{j}^{ \pm}(j=1,2)$ are neutralinos and charginos respectively. Let us first review the radiative two body decays of the gluino, which occur via quark-squark loop diagrams (figure 1$)$. The neutralino $\tilde{\chi}_{i}^{0}$ is an admixture of wino- $\left(\widetilde{W}_{3}\right)$, bino- $(\widetilde{B})$ and higgsino-like $(\widetilde{H})$ neutral spinors, which are respectively the superpartners of the neutral gauge eigenstates $B, W_{3}$ and of the two Higgs doublet of the MSSM $H_{u, d}$,

$$
\tilde{\chi}_{i}^{0}=N_{i 1} \widetilde{B}+N_{i 2} \widetilde{W}_{3}+N_{i 3} \widetilde{H}_{d}+N_{i 4} \widetilde{H}_{u}
$$

If the squarks are very heavy, from an effective Lagrangian point of view the decay width into a wino is strongly suppressed since it is induced by a dimension 7 operator [41, 42], whereas for a bino or a higgsino it can be induced already by a dimension 5 chromo-magnetic interaction,

$$
\mathcal{L}_{\text {eff. }}=\frac{1}{\widetilde{m}} \overline{\tilde{\chi}_{i}^{0}} \sigma^{\mu \nu} P_{L, R} \tilde{g}^{a} G_{\mu \nu}^{b} \delta_{a b}
$$




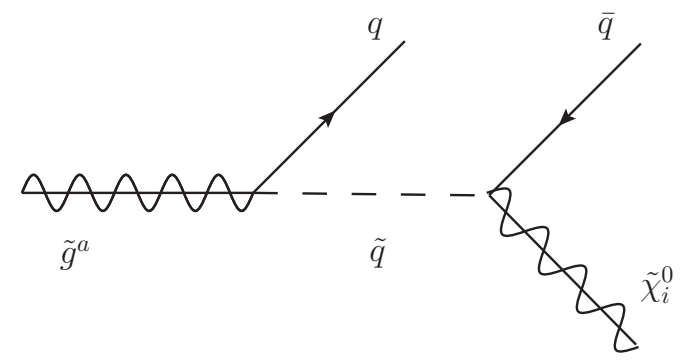

Figure 2. Three-body decay of the gluino where the (s)quark flavour runs through $q=u, d, c, s, t, b$.

where $\widetilde{m}$ is an effective squark mass scale. The partial widths of these two body-decays are given by $[35,41,42,66]$, in the heavy squark limit,

$\Gamma(\tilde{g} \rightarrow g \widetilde{B}) \simeq \frac{\alpha \alpha_{s}^{2}}{512 \pi^{2} c_{W}^{2}} \frac{\left(m_{\tilde{g}}^{2}-m_{\widetilde{B}}^{2}\right)^{3}}{m_{\tilde{g}}^{3}}\left[\sum_{q} \frac{Y_{q_{L}}}{m_{\widetilde{q}_{L}^{2}}^{2}}-\frac{Y_{q_{R}}}{m_{\widetilde{q}_{R}^{2}}^{2}}\right]^{2}\left(m_{\tilde{g}}-m_{\widetilde{B}}\right)^{2}$

$\Gamma(\tilde{g} \rightarrow g \widetilde{H}) \simeq \frac{\alpha \alpha_{s}^{2} m_{t}^{2}}{128 \pi^{2} M_{W}^{2} s_{W}^{2} s_{\beta}^{2}} \frac{\left(m_{\tilde{g}}^{2}-m_{\widetilde{H}}^{2}\right)^{3}}{m_{\tilde{g}}^{3}}\left[\frac{m_{t}}{m_{\tilde{t}_{L}}^{2}}\left(\ln \frac{m_{\tilde{t}_{L}}^{2}}{m_{t}^{2}}-1\right)+\frac{m_{t}}{m_{\tilde{t}_{R}}^{2}}\left(\ln \frac{m_{\tilde{t}_{R}}^{2}}{m_{t}}-1\right)\right]^{2}$

where $\alpha$ and $\alpha_{s}$ are the electromagnetic and strong couplings constants respectively. We used the short-hand notations $c_{W} \equiv \cos \theta_{W}, s_{W} \equiv \sin \theta_{W}, s_{\beta} \equiv \sin \beta$. The angle $\theta_{W}$ is the weak mixing angle and $\beta$ is defined through the ratio $\tan \beta=v_{u} / v_{d}$ where $v_{u, d}$ are the vacuum expectation values of the two $\mathrm{SU}(2)_{L}$ Higgs doublets $H_{u, d}$ of the MSSM. Both angles lie between 0 and $\pi / 2 . Y_{q}$, appearing in eq. (2.6), is the quarks hypercharge where the index $q$ runs through all flavours. In eq. (2.7), since the decay width is proportional to the top mass, only the stops-tops loops were kept. From eq. (2.6) we can see that $\Gamma(\tilde{g} \rightarrow g \widetilde{B})$ scales like $m_{\tilde{q}}^{-4}$ whereas $\Gamma(\tilde{g} \rightarrow g \widetilde{H})$ benefits from a logarithmic enhancement. The origin of this logarithmic enhancement can be understood from an effective Lagrangian point of view as follows. In the effective theory where the squarks have been integrated out, this decay is induced by a top-top-gluino-higgsino operator in which the two top quarks form a loop which can emit a gluon to form the chromo-magnetic gluino-gluonhiggsino interaction of eq. (2.5). Such a diagram is divergent in the effective theory thereby generating a logarithmic enhancement [35, 41], which is cut-off by the squark mass (the scale of the effective theory breakdown). Therefore this decay is an interesting probe of the scalar mass scale in case they are kinematically inaccessible from colliders [42]. In the very heavy squark limit the leading logarithmic corrections should be resummed to obtain reliable predictions using renormalisation-group techniques as has been done in [41].

Let us now turn on to the three body decays of the gluino $\tilde{g} \rightarrow q \bar{q} \tilde{\chi}^{0}$ which is mediated by squark exchange $\tilde{q}$ (figure 2), with the assumption that the squark sector respects flavour symmetries. In the massless quark limit, only the bino-like contributes and we can get an 
analytical expression of the gluino partial decay width [38, 41, 42, 66],

$$
\Gamma\left(\tilde{g} \rightarrow q_{L} q_{R}^{c} \widetilde{B}\right)=\frac{\alpha \alpha_{s} Y_{q_{L}}}{96 \pi \cos ^{2} \theta_{W}} \frac{m_{\tilde{g}}^{5}}{m_{\tilde{q}_{L}}^{4}}\left[f\left(\frac{m_{\widetilde{B}}^{2}}{m_{\tilde{g}}^{2}}\right)+\frac{2 m_{\widetilde{B}}}{m_{\tilde{g}}} g\left(\frac{m_{\widetilde{B}}^{2}}{m_{\tilde{g}}^{2}}\right)\right]
$$

where $f(x)=1-8 x-12 x^{2} \ln x+8 x^{3}-x^{4}$ and $g(x)=1+9 x+6 x \ln x-9 x^{2}+6 x^{2} \ln x-x^{3}$. Like the two-body decay into the bino-like neutralino in eq. (2.6), the three-body decay width scales as $m_{\tilde{q}_{L}}^{-4}$ in the heavy squark limit. If the first two squarks generations are decoupled from the third one, which is quite easily achieved in SUSY since the large top Yukawa usually drives the running of third generation squark masses to lighter value than the two others, the quarks produced in the three body decays will be composed of $t \bar{t}, b \bar{b}$ pairs. For generic quark masses the integration over the three-body phase space cannot be performed analytically and one must resort to a numerical integration. However, keeping only the quark mass dependence in the couplings and in the limits $m_{\tilde{\chi}^{0}} \ll m_{\tilde{g}}$ and $m_{\tilde{g}} \ll m_{\tilde{q}_{L}}, m_{\tilde{q}_{R}}$, the decay rate $\tilde{g} \rightarrow q \bar{q} \tilde{\chi}_{i}^{0}[36]$ is given by

$$
\Gamma\left(\tilde{g} \rightarrow q \bar{q} \tilde{\chi}_{i}^{0}\right) \simeq \frac{\alpha_{s} m_{\tilde{g}}^{5}}{384 \pi^{2}} \sum_{q}\left[\frac{\left|a_{q}\right|^{2}+\left|b_{q_{L}}\right|^{2}}{m_{\tilde{q}_{L}}^{4}}+\frac{\left|a_{q}\right|^{2}+\left|b_{q_{R}}\right|^{2}}{m_{\tilde{q}_{R}}^{4}}\right]
$$

The sum is over all quark species allowed by kinematics. The $a, b, c$ coefficients are given by

$$
\begin{aligned}
a_{u} & =\frac{g m_{u} N_{i 4}}{2 M_{W} \sin \beta}, & a_{d} & =\frac{g m_{d} N_{i 3}}{2 M_{W} \cos \beta}, \\
b_{q_{L}} & =g\left(\frac{Y_{q_{L}}}{2} \tan \theta_{W} N_{i 1}+T_{3}^{q_{L}} N_{i 2}\right), & b_{q_{R}} & =g Q_{q} \tan \theta_{W} N_{i 1}
\end{aligned}
$$

where $u \equiv u, c, t$ and $d \equiv d, s, b$. The electric charge $Q_{q}$ is defined as $Q_{q}=T_{3}^{q_{L}}+Y_{q} / 2$. It is then natural to ask oneself in which cases the two-body decay can dominate over the three-body one. To investigate this we define the ratio $R_{2 / 3}$ as

$$
R_{2 / 3}=\frac{\Gamma\left(\tilde{g} \rightarrow g \tilde{\chi}_{i}^{0}\right)}{\Gamma\left(\tilde{g} \rightarrow q \bar{q} \tilde{\chi}_{i}^{0}\right)}
$$

Obviously, the three-body decay is suppressed by the phase space when the mass difference between the gluino and the neutralino is too small. This is particularly true for $\tilde{g} \rightarrow t \bar{t} \tilde{\chi}_{i}^{0}$ when the $t \bar{t}$ threshold is closed, i.e $m_{\tilde{g}}-m_{\tilde{\chi}_{i}^{0}}<2 m_{t}$. In this situation the decay rates for $\tilde{g} \rightarrow b \bar{b} \tilde{\chi}_{i}^{0}$ may still compete with $\Gamma\left(\tilde{g} \rightarrow g \tilde{\chi}_{i}^{0}\right)$. In the higgisno-like case, the $b \bar{b}$ and $g \tilde{\chi}_{i}^{0}$ final states are dominated by the heaviest quark: the former is proportional to $m_{b}^{2}$ and the latter to $m_{t}^{4}$. In the limit where the left and right squark masses are equal for each respective flavour $q$, i.e $m_{\tilde{q}_{L}}=m_{\tilde{q}_{R}}=\tilde{m}_{q}$, using eqs. (2.7) and (2.9) and defining $x_{t}=m_{t}^{2} / \tilde{m}_{t}^{2}$, one finds for $R_{2 / 3}$ [36]

$$
R_{2 / 3}=\frac{24 \alpha_{s}}{\pi}\left(\frac{\tilde{m}_{b}}{\tilde{m}_{t}}\right)^{4}\left(\frac{m_{t}^{2}}{m_{\tilde{g}} m_{b} \tan \beta}\right)^{2}\left[\frac{1}{1-x_{t}}+\frac{\ln x_{t}}{\left(1-x_{t}\right)^{2}}\right]^{2}
$$

In the bino-like case, we would obtain a similar formula using eqs. (2.6) and (2.9), but $R_{2 / 3}$ only benefits from the parametric enhancement $\tilde{m}_{b} / \tilde{m}_{t}$. Hence, there are two conditions for which the two-body decay will dominate over the three-body one: 
- If the $t \bar{t}$ threshold is closed one needs a somewhat hierarchy between the sbottoms and the stops,

- Decouple all the squarks at very high masses, in this case $R_{2 / 3} \propto m_{t}^{2} / m_{\tilde{g}}^{2}\left[1+\ln x_{t}\right]^{2}$.

However, as stressed in [41], for such very heavy squarks, the logarithmic corrections have to be resummed and in the case of all squarks masses being equal and large, the resummation tends to decrease $R_{2 / 3}$ compared to eq. (2.13) when the common scalar mass scale $\tilde{m} \gtrsim 10^{6} \mathrm{GeV}$. On the other hand, in the bino-like case, $R_{2 / 3}$ does not benefit from such parametric enhancements. The lack of logarithmic enhancement for the two-body decay (i.e the higgsino-like neutralino is not allowed in gluino decays) implies that the three-body will generally dominate instead, if available.

It is worth adding that in the pure higgsino case, it is rather another three-body decay which rival with the gluino loop decay, namely $\tilde{g} \rightarrow t \bar{b} / \bar{t} b \tilde{\chi}_{1}^{ \pm}$. This is due to the fact that the neutral and charged higgsinos are almost degenerated in mass, and this decay will dominate only once the $t \bar{b} / \bar{t} b$ threshold is crossed, i.e $m_{\tilde{g}}-m_{\tilde{\chi}_{1}^{0}} \simeq m_{\tilde{g}}-m_{\tilde{\chi}_{1}^{ \pm}}>m_{t}+m_{b}$. These three-body decays do not suffer from a hierarchical third generation squark spectrum since they are mediated by stops. In the pure bino case this decay will be effective at larger $\Delta M$ since the lightest chargino $\tilde{\chi}_{1}^{ \pm}$is much heavier than the bino-like LSP.

In order to illustrate to which extent the radiative gluino decay can dominate over the other decay modes, without assuming any specific SUSY-breaking pattern, we performed a numerical scan within the pMSSM parameter space using SUSY-HIT [83]. In this numerical investigation we set all soft masses (sleptons and squarks) to $5 \mathrm{TeV}$ and trilinear couplings $A_{i}$ to zero except for the third generation squarks for which we chose the following range:

- $M_{\tilde{t}_{R}}=1 \mathrm{TeV}, M_{\tilde{Q}_{3}}=2 \mathrm{TeV}, A_{b}=0$

- $1 \mathrm{TeV}<M_{\tilde{b}_{R}}<2 \mathrm{TeV}$

- $-2 \mathrm{TeV}<A_{t}<2 \mathrm{TeV}$

The parameters related to the gaugino and Higgs sector were set to

- $400 \mathrm{GeV}<M_{1}, \mu<800 \mathrm{GeV}$

- $M_{2}=2 \mathrm{TeV}, M_{3}=600 \mathrm{GeV}, \tan \beta=10$

The scalar mass scale chosen is sufficiently low such that we ensure that the gluino would decay in the detector. We picked up only points giving the SM-like Higgs mass in the range $122 \mathrm{GeV}<m_{h}<128 \mathrm{GeV}$ and the resulting gluino mass is driven around $m_{\tilde{g}} \simeq 810 \mathrm{GeV}$ due to radiative corrections. We ensured that $m_{\tilde{g}}>m_{\tilde{\chi}_{1}^{0}}$ such that the lightest neutralino is always the LSP. We divided the surviving points of the scan into three different regions, depending on the nature of the lightest neutralino, whether it is mostly higgsino-like, binolike or a mixed bino-higgsino. The higgsino-like (bino-like) neutralinos correspond to a higgsino (bino) fraction greater than 90\%. The remaining points are an admixture of bino-higgsino like neutralinos which we dubbed as "mixed". 

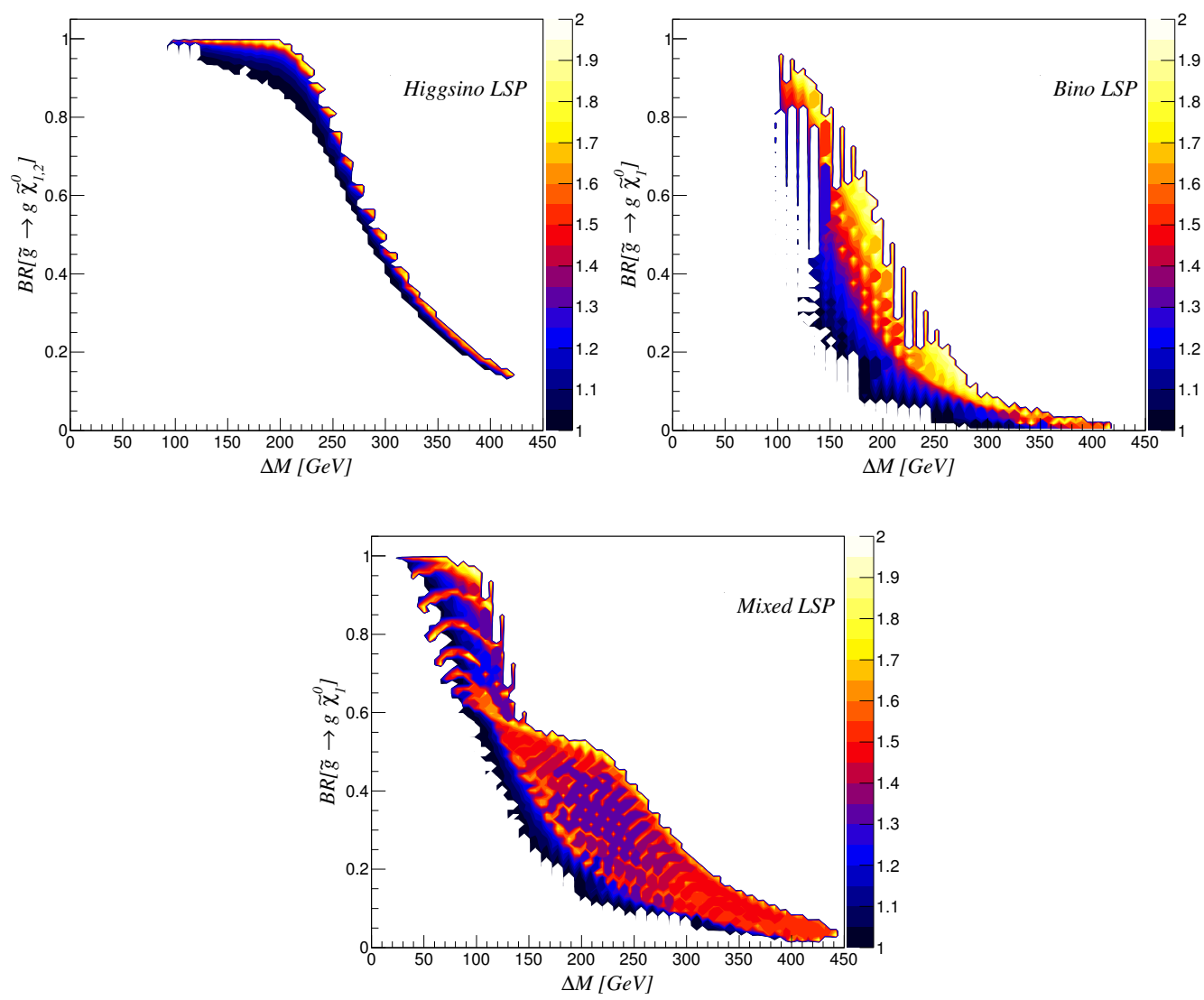

Figure 3. Branching ratio of $\tilde{g} \rightarrow g \tilde{\chi}_{1}^{0}$ in terms of $\Delta M=m_{\tilde{g}}-m_{\tilde{\chi}_{1}^{0}}$ for a higgsino-like (top,left), bino-like (top,right) and mixed bino-higgsino (bottom) neutralino. In the higgsino-like case the branching ratio of the radiative decay $\tilde{g} \rightarrow g \tilde{\chi}_{2}^{0}$ is also included. The colour shading indicates the ratio $m_{\tilde{b}_{1}} / m_{\tilde{t}_{1}}$.

We display in figure 3 the branching ratio of the two body decay of the gluino into a gluon plus the lightest supersymmetric particle (LSP) in terms of the mass difference $\Delta M=m_{\tilde{g}}-m_{\tilde{\chi}_{1}^{0}}$. Given the nature of the spectrum, only the radiative decays $\tilde{g} \rightarrow g \tilde{\chi}_{i}^{0}$ and the three-body modes into heavy quarks $\left(\tilde{g} \rightarrow b \bar{b} / t \bar{t} \tilde{\chi}_{i}^{0}\right.$ and $\left.\tilde{g} \rightarrow t \bar{b} / \bar{t} b \tilde{\chi}_{1}^{ \pm}\right)$are allowed. The colour shading correspond to the level of hierarchy between the lightest stop $\widetilde{t}_{1}$ and lightest sbottom $\widetilde{b}_{1}$ given by the ratio $m_{\widetilde{b}_{1}} / m_{\widetilde{t}_{1}}$. It is worth noting that in the higgsino-like case we also included the two-body decay of the gluino to the next-to-lightest neutralino $\tilde{g} \rightarrow g \tilde{\chi}_{2}^{0}$ since the mass difference between the $\tilde{\chi}_{1}^{0}$ and $\tilde{\chi}_{2}^{0}$ is at most $\simeq 15 \mathrm{GeV}$. For such a small mass difference the next-to-lightest neutralino $\tilde{\chi}_{2}^{0}$ would decay into the LSP and soft leptons or quarks originating from the off-shell $\mathrm{Z}$ boson decay in $\tilde{\chi}_{2}^{0} \rightarrow Z^{*} \tilde{\chi}_{1}^{0}$. Hence the channel $\tilde{g} \rightarrow g \tilde{\chi}_{2}^{0}$ would leave the same experimental signature of jets + MET in the detector as $\tilde{g} \rightarrow g \tilde{\chi}_{1}^{0}$. Glancing at figure 3 it is clear that largest branching fractions for the radiative decay are obtained when the mass difference $\Delta M$ is small. As one could expect from the approximate formulas in eqs. (2.6), (2.7), the branching ratio of the two body decays $\tilde{g} \rightarrow g \widetilde{H}_{1,2}$ (top, left panel of figure 3 ) gives the largest branching fractions. This is 

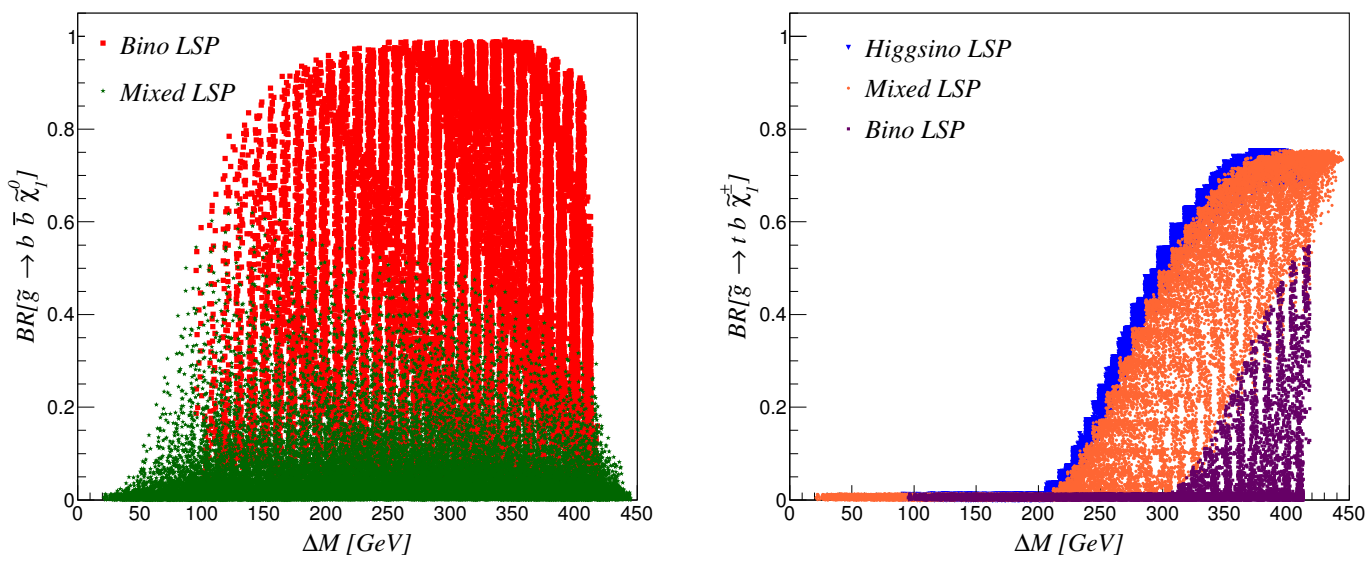

Figure 4. Branching ratios for the most important three-body decay modes depending on the composition of the LSP. The left panel corresponds to the channel $\tilde{g} \rightarrow b \bar{b} \tilde{\chi}_{1}^{0}$, where red squares correspond to the case where the LSP is bino-like and green stars when it is a mixed bino-higgsino. The pure higgsino case is not shown as it is negligible. The right panel displays the $\tilde{g} \rightarrow t \bar{b} / \bar{t} b \tilde{\chi}_{1}^{ \pm}$for each $\tilde{\chi}_{1}^{0}$ composition, blue diamonds points corresponds to situations where the LSP is higgsino-like, orange points and when it is mixed magenta squares.

due to the fact that it depends on the fourth power of the top mass, unlike the bino-like and mixed cases, see eqs. (2.6), (2.7). In addition, the dependence on ratio $m_{\widetilde{b}_{1}} / m_{\widetilde{t}_{1}}$ of the loop-induced decay is much more pronounced in the bino-like and mixed case than in the higgsino case. It is particularly true for the bino case (top, right panel) where largest branching fraction are only obtained when the hierarchy between the sbottoms and stops is significant. Moreover, the two-body decay into higgsino-like neutralinos dominate even for quite large $\Delta M$ : the radiative decay starts to fall off at $\Delta M \simeq 200 \mathrm{GeV}$, while for the bino-like and mixed cases they already decline at $\Delta M \simeq 100 \mathrm{GeV}$. The drops of the loop-induced decays can be understood from looking at figure 4 . In this figure are displayed the branching fractions of the most important three-body decays for each nature of the LSP. The left panel features the branching ratio $\operatorname{BR}\left(\tilde{g} \rightarrow b \bar{b} \tilde{\chi}_{1}^{0}\right)$ for bino-like or mixed neutralinos. The higgsino-like LSP case is not shown since it is negligible and buried in the two others. The right panel presents the branching fraction of the gluino decay into $t \bar{b} / \bar{t} b$ final states for all three LSP natures considered. In the higgsino case (blue diamonds in the right panel of figure 4), a comparison with the top, left panel of figure 3 makes it clear that as soon as the decay into $t \bar{b} / \bar{t} b$ is kinematically allowed, the branching fraction $\operatorname{BR}\left(\tilde{g} \rightarrow g \tilde{\chi}_{1,2}^{0}\right)$ decreases fast (for $\Delta M \gtrsim 200 \mathrm{GeV}$ ) and the three-body mode $\tilde{g} \rightarrow t \bar{b} / \bar{t} b \tilde{\chi}_{1}^{ \pm}$starts to take over. However, thanks to the logarithmic enhancement, the loop-decay of the gluino to neutral higgsinos still persists at large $\Delta M$ and is of order $10-20 \%$. The rationale for this decay mode to take over in the higgsino case is that, as soon as the threshold is open, it does not suffer from large suppression factors. Indeed, this decay is also mediated by stop exchange, as the loop decay is, and not suppressed by small quark masses. In the pure bino case, $\operatorname{BR}\left(\tilde{g} \rightarrow t \bar{b} / \bar{t} b \tilde{\chi}_{1}^{ \pm}\right)$is suppressed until larger 
$\Delta M$. This is due to the parameter range we chose for the scan. Indeed, for this decay mode to be open one needs a significant mass splitting between the gluino and the lightest chargino, typically $m_{\tilde{g}}-m_{\tilde{\chi}_{1}^{ \pm}} \gtrsim m_{t}+m_{b}$. In our scan $m_{\tilde{\chi}_{1}^{ \pm}}$is driven by the value of the parameter $\mu$, which must still be quite large to keep the LSP bino-like. Therefore one needs to reach a significantly larger mass splitting $\Delta M$ to fulfil these requirements, compared to the pure higgsino case where $m_{\tilde{\chi}_{1}^{0}} \simeq m_{\tilde{\chi}_{1}^{ \pm}} \simeq \mu$. Instead, the three-body decay mode $\tilde{g} \rightarrow b \bar{b} \tilde{\chi}_{1}^{0}$ quickly dominates over $\tilde{g} \rightarrow g \tilde{\chi}_{1}^{0}$, already from $\Delta M \simeq 100 \mathrm{GeV}$. This occurs even when the ratio $m_{\tilde{b}_{1}} / m_{\tilde{t}_{1}}$ is large. Again, the underlying argument is that the gluino loop decay to a bino-like neutralino does not profit from any enhancement, apart from phase space considerations. For a mixed neutralino, when $\Delta M$ increases, the suppression of the radiative decay is counter-balanced by both $\tilde{g} \rightarrow b \bar{b} \tilde{\chi}_{1}^{0}$ and $\tilde{g} \rightarrow t \bar{b} / \bar{t} b \tilde{\chi}_{1}^{ \pm}$.

Having identified in which region of parameter space of the pMSSM the radiative decay can dominate, we now turn on to which extent it can be constrained by existing ATLAS and CMS $8 \mathrm{TeV}$ analyses.

\section{Constraining the gluino loop decay from existing $8 \mathrm{TeV}$ LHC analyses}

It is expected that the typical signature of the radiative gluino decay will lead to a preponderance of jets. If favoured, when the mass difference $\Delta M=m_{\tilde{g}}-m_{\tilde{\chi}^{0}}$ is small, the final state jets are typically as soft as those originating from the parton shower and in turn the gluino decay gets buried into the huge QCD background. To increase the sensitivity one can resort to, similarly to what is done concerning Dark Matter or compressed spectra searches, trigger on a hard initial-state-radiation (ISR) jet, the so-called monojet searches.

Since the radiative gluino decay has not been fully investigated at the LHC, we wish to fill this gap, using in particular monojet analyses to be sensitive to it. It is worth to note that the multijet analysis performed in [6] has indeed probed a small mass $\Delta M$. Nevertheless, the interpretation has been done in a different context from ours, where the first and second generation squarks are not too heavy and therefore the three-body decay into light-flavour quarks $\tilde{g} \rightarrow q \bar{q} \tilde{\chi}_{1}^{0}$ is not suppressed. Thus, one can probe small mass splitting between the gluino and the LSP in this case. In [6], the direct decay of the gluino to a gluon and a neutralino was also used to constrain $m_{\tilde{g}}$ and $m_{\tilde{\chi}_{1}^{0}}$, but based only on a fully hadronic search. Only numbers for particular values of $m_{\tilde{g}}$ and $m_{\tilde{\chi}_{1}^{0}}$ were presented. For a massless neutralino, gluino masses below $1250 \mathrm{GeV}$ can be excluded, and a lower mass limit of $m_{\tilde{\chi}_{1}^{0}}=550 \mathrm{GeV}$ corresponding to $m_{\tilde{g}}=850 \mathrm{GeV}$ is quoted, but no full coverage of the $m_{\tilde{g}}-m_{\tilde{\chi}_{1}^{0}}$ mass plane has been presented. In [28], an exclusion contour in the $m_{\tilde{g}}-m_{\tilde{\chi}_{1}^{0}}$ mass plane has been derived using monojet searches, using the process $\tilde{g} \rightarrow q \bar{q} \tilde{\chi}_{1}^{0}$ assuming $100 \%$ branching fraction, among other scenarios investigated. However, as we saw in the previous section, the dominance of this channel strongly depends on the squark spectrum, which is not detailed in [28], except that the stops and sbottoms are assumed to be light. We saw in the previous section that the radiative decay is favoured when light stops are present and therefore it is not clear if the assumption that $\tilde{g} \rightarrow q \bar{q} \tilde{\chi}_{1}^{0}$ dominates holds.

In the context where the first and second generation of squarks are degenerate, bounds on their masses are pushed beyond the $\mathrm{TeV}$ scale. In the scenario we are focussing on, 
namely with decoupled first and second generations with somewhat lighter third generation, this effort is complementary to the official experimental analyses and worth to investigate. Achieving such a task then requires to recast the already existing ATLAS and CMS analyses since we do not have access to the raw data of both collaborations. The experimental signature for the gluino radiative decay is rather simple: it only consists of jets (possibly very soft) and missing transverse energy (MET). A characteristic feature of scenarios where the two-body radiative gluino decay dominate is typically the ones which are difficult to probe in a collider environment, namely ones where the mass difference between the mother and daughter particles is small (i.e compressed spectra). These scenarios are difficult to explore since they lead to final states with less energetic states or leptons, thus reducing the detection efficiency and signal acceptance. This may be part of the reasons why this decay pattern has not been fully considered as worth to investigate first in the past, as a priori its prospects are not very enthusiasming. In addition, in typical mSUGRA/CMSSM scenarios, this branching ratio is quite small since the mass splitting between the gluino of the LSP is generically quite large. However, the advent of monojet searches to precisely increase the sensitivity to compressed spectra has been a game changer in this respect. As noted in [42], when the ratio of the Higgs doublet vev's $\tan \beta \simeq \mathcal{O}(1)$, a $125 \mathrm{GeV}$ Higgs implies a non-negligible branching fraction of the radiative modes for the gluino decay. Moreover it is well-motivated in SUSY scenarios where the gluino is the next-to-lightest supersymmetric particle (NLSP), the so-called GNLSP models. Examining this process at the LHC can also put constraints on the viability of these models in solving the Dark Matter problem. Indeed, in such models, the right amount of relic density is obtained through important coannihilation processes between the LSP and the gluino and their efficiency is mainly driven by $\Delta M$ see for example, [84] and references therein. Hence, a full quantitative investigation of the radiative gluino at the LHC seems timely.

The experimental signature of the radiative decay can be categorised in 1,2 and 3 jets bins plus MET. Unfortunately there exists no published official analysis covering all such categories. In this purpose, and to cover all jet bins, we reimplemented within the MadAnalysis5 [80, 81] framework the following three analyses:

- ATLAS-SUSY-2013-21 [85]: this analysis targets the stop decay $\widetilde{t}_{1} \rightarrow c+\tilde{\chi}_{1}^{0}$ using a monojet analysis. It has also been used to probe the small squark-neutralino mass difference using $\tilde{q} \rightarrow q+\tilde{\chi}_{1}^{0}$, see [6],

- ATLAS-SUSY-2013-02 [86]: search for squarks and gluinos in final states using high$p_{T}$ jets with no leptons, 2-6 jets and large MET,

- CMS-SUS-13-012 [87]: search for new physics in multijet events with large MET divided into three jet multiplicity categories $(3-5,6-7$, and 8 jets),

in order to work out the $8 \mathrm{TeV}$ LHC constraints using $20.3 \mathrm{fb}^{-1}$ of collected data. The recasted CMS-SUS-13-012 SUSY search was already available in the MadAnalysis5 Public Analysis Database [82] and can be found at [88]. The two remaining searches were implemented and validated for the present work and are now available at [89, 90]. For the sake of clarity, we present in the appendix A a comparison between the official cut flows 
and our reimplementations of the ATLAS monojet [85] and multijet [86] analyses within MadAnalysis5 (MA5). From tables 4, 7 and 8 the selection cuts of each respective analysis can be read off.

For the monojet search, see table 4, one can see that the agreement is not very good at the level of the preselection cuts. The discrepancy originates already from the $E_{T}^{\text {miss }}>$ $100 \mathrm{GeV}$ cut applied at the reconstructed level. The MET trigger efficiency varies with $E_{T}^{\text {miss }}$ and in particular is not $100 \%$ effective at $E_{T}^{\text {miss }}=100 \mathrm{GeV}$. To reproduce the MET trigger efficiency we parametrised the efficiency turn-on curve presented in [91] coming from the ATLAS simulation of the process $p p \rightarrow Z H \rightarrow \nu \bar{\nu} b \bar{b}$, as advised after communication with the ATLAS SUSY conveners. We already observe at this level a discrepancy which ranges from $16 \%$ to $32 \%$ for the three benchmark points between our implementation and the ATLAS result. Moreover we cannot reproduce the Trigger, Event Cleaning, and Bad Jet veto efficiencies, as we do not have access to this information in official documentation. However, once we enter the signal regions, the relative efficiencies of our cuts are comparable with the official ones and at the level of final number of events we observe an agreement between 15-25\%, which is in the ballpark of expected accuracies from fast-simulation. More details about the validation of the recasting procedure can be found at [92].

For the ATLAS multijet analysis [86] a cut flow comparison is also presented in tables 7 and 8. One can see that the agreement between the recasted analysis and the official is fairly good. The CMS multijet implementation and validation was already available on the PAD [82] before the initiation of this work and all details were presented there.

As official results are not provided in terms of the gluino branching ratios and the radiative mode has not been fully investigated so far, we derived limits on this decay pattern within a Simplified Model approach. In the case we focussed only the gluino and LSP are relevant in the mass spectrum and we assume $\operatorname{BR}\left(\tilde{g} \rightarrow g \tilde{\chi}_{1}^{0}\right)=100 \%$. The signal generation was done using MADGRAPH5 [93, 94] using the CTEQQ6L1 parton distribution functions [95], as

$$
p p \rightarrow \tilde{g} \tilde{g}+X \rightarrow j j+E_{T}^{\text {miss }}
$$

where $X=0,1$ is the number of generated jets in the signal sample. The MADGRAPH samples are produced using the AUET2B tune $[96,97] .{ }^{1}$ The MLM [99] matching scheme is used, and a MADGRAPH $k_{T}$ measure cut-off and a PYTHIA [100] jet measure cut-off both set to 0.25 times the mass scale of the SUSY particles produced in the hard process. Hadronisation is performed using PYTHIA-6.426 [100] as incorporated in the MADGRAPH-5 package and detector effects are simulated with the 'MA5tune' of DELPHES-3 [101] as

\footnotetext{
${ }^{1}$ It is worth to note that care should be taken when using recent versions of MadGraph5 together with the AUET2B tune. Indeed this tune was designed from a Leading-Order (LO) Matrix Element + Parton Shower (MEPS) merging. Since MadGraph5.2, the matching MEPS is done at next-to-leading order and in the same fashion as in MCNLO [98]. Therefore if one uses this tune together with a version 5.2 or higher of MadGraph, the interpretation of the results should be taken with great care. Indeed, we observed significant discrepancies in reproducing the official exclusion curves between using a recent version of MadGraph or the one actually adopted in the official analyses in $[85,86]$. That is why, for each analysis, we stick as much as possible to the Monte-Carlo configurations described in the experimental analyses. Thus, each signal sample has been generated with a different MadGraph version for each analysis we recasted.
} 

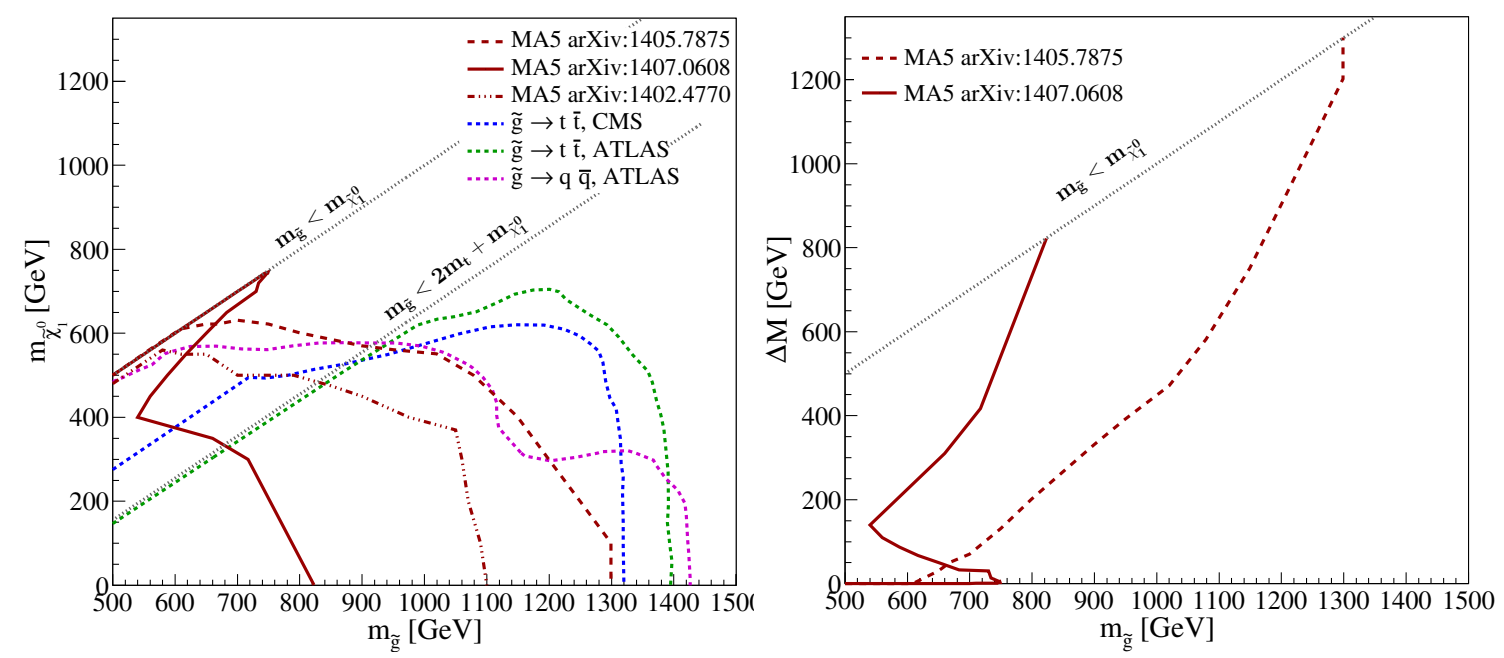

Figure 5. Left panel: 95\% CL exclusion contours for the radiative gluino decay simplified topology. The solid red line corresponds to the mass limits obtained from the MA5 recasted ATLAS monojet analysis [90], the red broken line from the MA5 recasted ATLAS multijet search [89] and the dasheddotted line from the MA5 recasted CMS multijet analysis [88]. For comparison, the official $95 \%$ CL exclusion lines for the $\tilde{g} \rightarrow t \bar{t} \tilde{\chi}_{1}^{0}$ SMS from ATLAS (green dashed line) [6] and CMS [22](blue dashed line) as well as for the $\tilde{g} \rightarrow q \bar{q} \tilde{\chi}_{1}^{0}$ SMS from ATLAS [6](purple dashed line) are also shown. Right panel: $95 \%$ CL exclusion contours in the plane $m_{\tilde{g}}$ versus $\Delta M$ where $\Delta M=m_{\tilde{g}}-m_{\tilde{\chi}_{1}^{0}}$. Only the exclusion contours from the MA5 recasted monojet and multijet analyses are given since they provide the best sensitivity in the low $\Delta M$ region.

described in [81]. The number of events are then normalised to the correct luminosity after including cross sections at the next-to-leading order and next-to-leading logarithm accuracy [102], as tabulated by the LHC SUSY Cross section Working Group [103]

For the statistical interpretation, we make use of the module exclusion_CLs.py provided in the MadAnalysis5 recasting tools. Given the number of signal, expected and observed background events, together with the background uncertainty (both directly taken from the experimental publications), exclusion_CLs. py determines the most sensitive signal region (SR), the exclusion confidence level using the $\mathrm{CL}_{s}$ prescription from the most sensitive SR, and the nominal cross section $\sigma_{95}$ that is excluded at 95\% CL. The simplified model parameter space is simply scanned by varying $m_{\tilde{g}}$ and $m_{\tilde{\chi}_{1}^{0}}$ until a $95 \%$ CL excluded number of BSM signal events is reached. The derived limits in the $\left(m_{\tilde{g}}, m_{\tilde{\chi}_{1}^{0}}\right)$ plane are presented in figure 5 . In the left panel of figure 5 the red lines are the $95 \%$ CL exclusion bounds using the recasted analysis. ${ }^{2}$ The results of the three recasted analyses (solid line: ATLAS monojet [90], broken line: ATLAS multijet [89] and dashed-dotted: CMS multijet [88]) are compared against three official published results: in green and blue are displayed the $95 \%$ CL limits obtained from the simplified topology $\tilde{g} \rightarrow t \bar{t} \tilde{\chi}_{1}^{0}$ from ATLAS [6] for the former and from CMS for the latter [22]. One immediately observes that the official SMS interpretations are limited when the $t \bar{t}$ threshold of the gluino three-body

\footnotetext{
${ }^{2}$ Note that one could have used, for the monojet analysis, the acceptance times efficiency tables as provided on the Twiki page of [85]. We chose to recast anyway this analysis to enrich the PAD [82].
} 
decay is closed. The CMS results pushes the limit a little bit above the $t \bar{t}$ threshold limit using off-shell tops, as compared to ATLAS. The purple broken line corresponds to the ATLAS limits in the $\left(m_{\tilde{g}}, m_{\tilde{\chi}_{1}^{0}}\right)$ mass plane for the $\tilde{g} \rightarrow q \bar{q} \tilde{\chi}_{1}^{0}$ simplified topology using the multijet search [6]. In the corresponding model investigated all squarks are decoupled and since the final states quarks are massless in this simplified approach, a small $\Delta M$ can be probed. In our scenario only the light-flavour squarks are completely decoupled from the low-energy spectrum and therefore our results are complementary to these.

A word of caution is in order before discussing and interpreting our results. Indeed, we do not provide an estimation of the theoretical uncertainties on both the signal cross section and acceptance. This certainly limits our ability to provide an unambiguous statement on which portion of the parameter space in the $\left(m_{\tilde{g}}, m_{\tilde{\chi}_{1}^{0}}\right)$ mass plane is excluded or not, but nevertheless provide a very interesting estimation of the sensitivity and mass reach of the $8 \mathrm{TeV}$ analyses we reimplemented. Nevertheless, comparing with the existing literature enabled us to estimate the correctness of our results. First of all, comparing with figure 4 of [28], we see that the shape of the monojet exclusion curve is similar and its maximum $\left(m_{\tilde{g}}, m_{\tilde{\chi}_{1}^{0}}\right)$ excluded values lie at $\left(m_{\tilde{g}}, m_{\tilde{\chi}_{1}^{0}}\right) \simeq(700,700) \mathrm{GeV}$, which is quite similar to our own maximum which sits at $\left(m_{\tilde{g}}, m_{\tilde{\chi}_{1}^{0}}\right) \simeq(750,750)$. In addition, the kink appearing for $\left(m_{\tilde{g}}, m_{\tilde{\chi}_{1}^{0}}\right) \simeq(540,400) \mathrm{GeV}$ is also observed in other interpretations of monojet analyses (see $[6,28]$ ). The underlying explanation of the kink comes from the fact that the monojet analysis is not, strictly speaking, based on a single hard jet. Indeed, the monojet selection cuts in [85] actually allow a number of jets $N_{\text {jets }} \leq 3$ with $p_{T}>30 \mathrm{GeV}$ and $|\eta|<2.8$. Thus the appearance of the kink is due to the fact that, starting from this point which possesses a sufficient $\Delta M$, additional jets fulfilling the above requirement populate the SRs, thereby enhancing the sensitivity. Next, comparing with the numbers given in figure 21 in [6], for a massless neutralino, gluino masses up to $m_{\tilde{g}}=1250 \mathrm{GeV}$ are excluded, whereas we exclude up to $m_{\tilde{g}}=1300 \mathrm{GeV}$ (see the broken red line in the left panel of figure 5 ). A lower mass limit of $m_{\tilde{\chi}_{1}^{0}}=550 \mathrm{GeV}$ for $m_{\tilde{g}}=850 \mathrm{GeV}$ is also quoted to be compared with $m_{\tilde{\chi}_{1}^{0}} \simeq 600 \mathrm{GeV}, m_{\tilde{g}}=850 \mathrm{GeV}$ on our side. Therefore our results are in fairly good agreement with the existing litterature. Last, there has been numerous studies, mostly in the context of Simplified Dark Matter models (see e.g [104, 105]), showing that the monojet searches can be sensitive to the decay width of the intermediate particles. However, since in our case the gluino decays through only one decay mode, its total width $\Gamma$ is suppressed and $\Gamma / m_{\tilde{g}} \ll 1$, such that we can use safely the narrow-width approximation implicitly assumed in our SMS approach.

Provided this important clarification, let us now discuss our results. As expected, the monojet analysis is the most sensitive search to probe the scenario where the gluino and neutralino are almost degenerate. Mass-degenerate gluino and LSP are excluded up to $750 \mathrm{GeV}$. As soon as $\Delta M$ increases, the efficiency of the monojet analysis quickly drops. This can be seen on the right panel of figure 5, where we display the two most sensitive recasted analyses in the small $\Delta M$ region. The colour coding is the same as in the left panel. The monojet analysis is the most effective in constraining a compressed gluino-neutralino spectrum up to $\Delta M \simeq 50 \mathrm{GeV}$. 
For larger $\Delta M$ the ATLAS multijet analysis becomes the most sensitive one in constraining the radiative decay. At low $m_{\tilde{g}}$ the signal region dubbed as $2 \mathrm{jl}$ ("2 loose jets", more details about the SR definition can be found in the official analysis [86]) drives the exclusion curve, at moderate $m_{\tilde{g}}$ it is driven by SR $2 \mathrm{jl}$ and $3 \mathrm{jm}$ ("3 medium jets") and at large $m_{\tilde{g}}$ the $4 \mathrm{jl}$ ("4 loose jets") SR dominates. Within this analysis gluino masses up to $m_{\tilde{g}} \simeq 1300 \mathrm{GeV}$ for a massless neutralino are excluded. It appears that the CMS multijet analysis is less powerful than the two ATLAS ones. There are two reasons for that, one is that the jet binning only starts from 3 jet bins and the second is that the search was designed as a discovery one, therefore its constraining power is less effective. Another comment is worth to be mentioned here: below the line where the $t \bar{t}$ threshold is open, interpreting the exclusion contour is highly debatable since we expect the three-body decays to take over. Therefore below this line our results should be taken with caution and the official results focussing on the three-body decay $\tilde{g} \rightarrow t \bar{t}$ are more realistic.

In the light of these results, it would be interesting to perform a combination of at least the two ATLAS analyses to get a more realistic exclusion curve. However, this goes beyond the scope of this paper since we do not have the necessary information at our disposal to perform the combination.

\section{Prospects at the $13 \mathrm{TeV}$ LHC}

We address the discovery and exclusion reach of the radiative gluino decay at $13 \mathrm{TeV}$ in this section. Since the radiative decay is dominant with a small mass gap between $\tilde{g}$ and $\widetilde{\chi}_{1,2}^{0}$, we design a special set of cuts optimized to investigate this case. To simplify matters we select benchmark points with a $\tilde{g}-\widetilde{\chi}_{1,2}^{0}$ SMS in mind, where the decay $\tilde{g} \rightarrow g \widetilde{\chi}_{1,2}^{0}$ is $100 \%$. As we already observed in section 2 , such an assumption is well motivated from theoretical considerations. We choose three representative benchmark points(BP) with nearly degenerate masses for $\tilde{g}$ and $\widetilde{\chi}_{1}^{0}$.

To achieve this we decouple the rest of the spectrum from this set, i.e we set the three generation of squarks and the sleptons to $3 \mathrm{TeV}$. Since a higgsino like scenario is favourable for the radiative decay, we set $M_{1}, M_{2}$ to $2 \mathrm{TeV}$, while $\mu$ is kept relatively light so that $\widetilde{\chi}_{1}^{0}, \widetilde{\chi}_{2}^{0}$ are Higgsino like. For all benchmark points, the mass gap $\Delta m=m_{\tilde{g}}-m_{\widetilde{\chi}_{1}^{0}}$ is fixed to around $10 \mathrm{GeV}$. The availability of phase space ensures that both $\tilde{g} \rightarrow g \widetilde{\chi}_{1}^{0}$ and $\tilde{g} \rightarrow g \widetilde{\chi}_{2}^{0}$ are equally important. However, since $\widetilde{\chi}_{1}^{0}$ and $\widetilde{\chi}_{2}^{0}$ are almost degenerate, the decay products of $\widetilde{\chi}_{2}^{0}$ are soft, and are most likely to go undetected.

The relevant masses and the branching ratios (BR) are tabulated in table 1 . The benchmark points were generated using SUSYHIT [83]. All the benchmark points satisfy the constraints derived in the previous section.

Since we focus on the nearly mass degenerate $\tilde{g}-\widetilde{\chi}_{1}^{0}$ scenario, the conventional search strategy is to look for a monojet + missing energy signature, where the hard ISR monojet recoils against the missing energy. However, at $13 \mathrm{TeV}$ the number of QCD radiation jets from the gluino can be fairly large and carry large transverse momenta. We argue in this section, that a dijet + missing energy scenario (from ISR/FSR) is better suited compared to the traditional monojet + missing energy search strategy. We argue that the number 


\begin{tabular}{|c|c|c|c|}
\hline & BP1 & BP2 & BP3 \\
\hline$M_{3}$ & 730 & 820 & 1100 \\
$\mu$ & 985 & 1180 & 1370 \\
\hline$m_{\tilde{g}}$ & 1005 & 1205 & 1405 \\
\hline$m_{\tilde{\chi}_{1}^{0}}$ & 995 & 1195 & 1395 \\
\hline$m_{\tilde{\chi}_{2}^{0}}$ & 1004 & 1201 & 1398 \\
\hline $\operatorname{BR}\left(\tilde{g} \rightarrow g \tilde{\chi}_{1}^{0}\right)(\%)$ & 99 & 53 & 47 \\
\hline $\operatorname{BR}\left(\tilde{g} \rightarrow g \tilde{\chi}_{2}^{0}\right)(\%)$ & 1 & 45 & 47 \\
\hline
\end{tabular}

Table 1. The masses and branching ratios for the three benchmark points used for the collider analysis.

of hard ISR jets can be significantly large at $13 \mathrm{TeV}$ LHC, and including a second ISR jet helps considerably in improving the signal to background ratio.

The principal backgrounds to this process are listed below,

- QCD: the QCD dijet events are the largest source of backgrounds in terms of cross section $\left(\sim 10^{8} \mathrm{pb}\right)$. A significant amount of missing energy is also likely to be present from detector mis-measurements.

- $\mathbf{t} \overline{\mathbf{t}}+$ jets: the multijet + missing energy topology is fairly common for all hadronic top decays. It is possible that the leptons and jets are missed which can lead to a dijet + missing energy topology. Since the $t \bar{t}+$ jets cross section is significantly large at $13 \mathrm{TeV}(\sim 900 \mathrm{pb})$, even a small efficiency after cuts can result in a significantly large background cross section.

- $\mathbf{Z}+$ jets: this constitutes the irreducible part of the background when the $\mathrm{Z}$ boson decays invisibly to a pair of neutrinos. Since the cross section is quite large $\left(\sim 10^{5} \mathrm{pb}\right)$, suppressing this background to a reasonable level is of primary requirement.

- ZZ: this background is similar to the $\mathrm{Z}+$ jets background, with one of the $\mathrm{Z}$ boson decaying hadronically while the other invisibly. This background is thus also one of the irreducible backgrounds for our case.

- $\mathbf{W}+$ jets: for a leptonic decay of $\mathrm{W}$, a missed lepton would mean a topology similar to the signal. This has to be therefore considered as a serious background for this topology.

- WZ: the WZ background can be significantly large for a hadronically decaying W and an invisibly decaying $\mathrm{Z}$.

Parton level events for the signal and background are generated using MADGRAPH5 . 0.7 [93], and passed on to PYTHIA6 [100] for showering and hadronisation. 

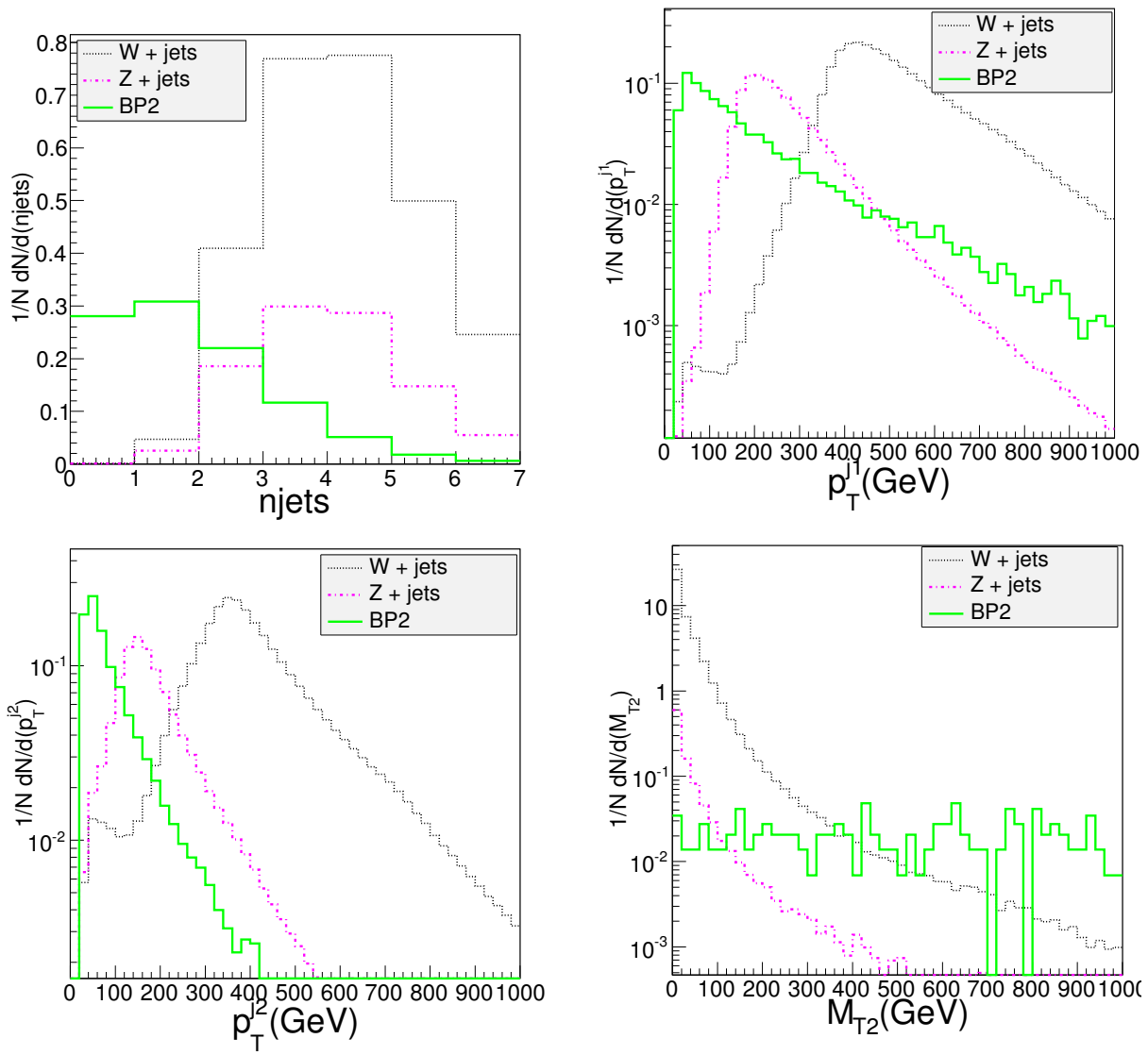

Figure 6. The distribution of the number of njets(top left), and the transverse momentum of the leading $\operatorname{jet}\left(p_{T}^{j_{1}}\right.$,top right) are presented in the top panel, while the transverse momentum of the second leading jet $\left(p_{T}^{j_{2}}\right.$, bottom left) and the $\mathrm{M}_{\mathrm{T} 2}$ (bottom right) distribution is plotted in the lower panel for the signal benchmark point BP2 and the $\mathrm{W} / \mathrm{Z}+$ jets backgrounds. The $M_{T 2}$ distribution is subject to all other cuts, as described in the text. The figures are normalized to unity.

For the signal additional two partons are generated at the matrix element level. For backgrounds like $t \bar{t}+$ jets, $\mathrm{W} / \mathrm{Z}+$ jets, additional 3 partons are generated in MADGRAPH5. The merging parameter for the matrix element-parton showering matching is set to $50 \mathrm{GeV}$. Since we anticipate that the bulk of the backgrounds are likely to originate from the tail of the $p_{T}$ spectrum we simulate $\mathrm{W} / \mathrm{Z}+$ jets with a hadronic energy $H_{T}\left(\right.$ defined as $\left.\Sigma p_{T}^{j}\right)$ cut of $400 \mathrm{GeV}$ at the parton level in MADGRAPH5. The sample is then passed on to Delphes3 [101] (specifically the MadAnalysis5 tune [81] ) for detector simulation. Jets are reconstructed using Fastjet [106] with an anti- $k_{T}$ [107] algorithm and a $p_{T}$ threshold of $30 \mathrm{GeV}$ and $\left|\eta_{j}\right| \leq 3$. Background cross sections are calculated using MADGRAPH5, while the gluino pair production cross section with decoupled sfermions are are quoted from the official SUSY cross section working group page [108]. We use the MadAnalysis5 [81] framework for the analysis of signal and background.

The following cuts are applied to maximize the signal to background ratio:

- Lepton Veto (C1): since the signal has no leptons, a lepton veto is imposed. Leptons are selected with a with transverse momentum threshold, $p_{T}^{l} \geq 10 \mathrm{GeV}$ and 
$|\eta| \leq 2.5$. Leptons are isolated by demanding that the scalar sum of the transverse momenta of all visible stable particles within a cone $\Delta R=0.2$, does not exceed $10 \%$ of $p_{T}^{l}$. This ensures that backgrounds with leptonic decays of $\mathrm{W} / \mathrm{Z}$ are suppressed.

- Jets (C2): we demand exactly two jets in the final state. From the plot of number of jets (njets) for the signal and background (top left figure 6), we observe that even with a small mass gap for the signal, it is possible to obtain up to 3 jets quite easily from QCD radiation. The background, especially, for the $\mathrm{W}+$ jets, peaks at 4 jets. Therefore it is convenient to design a search strategy with a dijet signature.

- b-jet veto (C3): to suppress the large $t \bar{t}$ background, we veto b-jets. This also ensures that backgrounds like $Z b \bar{b}, W b \bar{b}$ are suppressed.

- $\boldsymbol{p}_{T}^{j_{1}, j_{2}}>$ 600, $200 \mathrm{GeV}(\mathrm{C} 4)$ : from figure 6 we note that the leading ISR jet can be significantly energetic for the signal (top right, figure 6) and therefore we impose a hard cut on the transverse momenta of the leading jet. Although the second leading jet is not as energetic (bottom left, figure 6), the tail of the distribution can go up to about $400 \mathrm{GeV}$. We therefore impose a cut of $200 \mathrm{GeV}$ on the second leading jet. The hard cuts on the leading jets ensure that we are in a region where the missing transverse energy that recoil against these hard ISR jets is high.

- $\boldsymbol{M}_{\boldsymbol{T 2}}$ (C5): for this study, the variable $M_{T 2}[109-111]$ is defined as,

$$
M_{T 2}\left(j_{1}, j_{2}, \not p_{\mathrm{T}}\right)=\min \left[\max _{\mathrm{T}}\left(\mathrm{j} 1, \not p_{\mathrm{T}}^{1}\right), \mathrm{M}_{\mathrm{T}}\left(\mathrm{j} 2, \not p_{\mathrm{T}}^{2}\right)\right]
$$

where the minimization is performed over all possible partitions of the missing transverse momentum, $\not p_{\mathrm{T}}=\not p_{\mathrm{T}}^{1}+\not p_{\mathrm{T}}^{2}$, and $j_{1}, j_{2}$, represent the two leading jets. The transverse mass of the system is defined as

$$
M_{T}^{2}\left(j, \not p_{\mathrm{T}}\right)=\mathrm{M}_{\mathrm{j}}^{2}+\mathrm{M}_{\chi}^{2}+2\left(\mathrm{E}_{\mathrm{T}}^{\mathrm{j}} \mathrm{E}_{\mathrm{T}}^{\chi}-\tilde{\mathrm{p}}_{\mathrm{T}}^{\mathrm{j}} \cdot \tilde{\mathrm{p}}_{\mathrm{T}}^{\chi}\right)
$$

The value of $M_{T}$ is bounded by the parent particle. It can be shown that this holds true for $M_{T 2}$ as well, i.e, after minimization, $M_{T 2}$ is bounded by the mass of the parent particle. To suppress SM backgrounds, where the only source of missing energy are the neutrinos, the value of the mass of the invisible particle is set to 0 . SM backgrounds like WW, WZ, ZZ, $t \bar{t}$, are expected to be cut off at the parent particle mass, while since the gluino is massive it is expected to go up to higher values. Uncorrelated/ISR jets however can significantly alter the shape of the $M_{T 2}$ distribution [112]. For low mass differences, the extra ISR jets that dominate the jet configuration are not correlated with the $\not p_{\mathrm{T}}$, and hence the $M_{T 2}$ distribution extends well beyond the gluino mass. In the bottom left panel of figure 6 we present the $M_{T 2}$ distribution for the signal(BP2) and the $\mathrm{W} / \mathrm{Z}+$ jets background subject to all the previously imposed cuts. We observe that the $M_{T 2}$ distribution falls sharply for the background, while the signal remains fairly flat. We use an optimized cut of $800 \mathrm{GeV}$ to suppress the background. 


\begin{tabular}{|c|c|c|c|c|c|c|}
\hline & $m_{\tilde{g}}, m_{\tilde{\chi}_{1}^{0}}$ & C.S & $\mathrm{N}$ & $\mathrm{C} 1+\mathrm{C} 2+\mathrm{C} 3$ & $\mathrm{C} 4$ & $\mathrm{C} 5$ \\
\hline & $(\mathrm{GeV})$ & $\mathrm{fb}$ & & & & \\
\hline $\mathrm{BP} 1$ & 1005,999 & 314 & $100 \mathrm{~K}$ & 134 & 4.2 & 1.6 \\
$\mathrm{BP} 2$ & 1205,1195 & 83 & $100 \mathrm{~K}$ & 46 & 2.3 & 0.6 \\
BP3 & 1405,1395 & 24 & $100 \mathrm{~K}$ & 12 & 0.7 & 0.21 \\
\hline $\mathrm{Z}+$ jets & & $1.7 \times 10^{8}$ & $5 \mathrm{M}$ & 783250 & 12395 & 1.7 \\
\hline $\mathrm{W}+$ jets & & $5.8 \times 10^{8}$ & $5 \mathrm{M}$ & 31921.1 & 7793.1 & 1.0 \\
\hline
\end{tabular}

Table 2. The cut flow table for the signal and background for the analysis. For simplicity we only provide the processes which yielda non-negligible background contribution. The cross section after each cut is tabulated from column 5 .

We summarize our cut flows for signal and background in table 2. For simplicity, we only document the backgrounds which leave a non negligible contribution after all cuts. In table 2, the production cross section (C.S) for signal and background processes are tabulated in the third column in femtobarn. The number of simulated events $(\mathrm{N})$ are noted in the 4 th column. From the fifth column onwards the cumulative effect of cuts are presented.

From table 2, we observe that the cut on the transverse momenta of the leading jets (C4), and $M_{T 2}$ (C5) are most effective in suppressing $\mathrm{W} / \mathrm{Z}+$ jets background. The other backgrounds (not tabulated) like QCD, $t \bar{t}+$ jets, WW,WZ,ZZ are reduced to a negligible level after the application of the $M_{T 2}$ cut. Although the signal process suffers as well as a result of the $M_{T 2}$ cut, the signal to background ratio is enhanced considerably. After all cuts, the total background cross section is reduced to $2.7 \mathrm{fb}$, while the signal cross sections vary from $1.6 \mathrm{fb}$ for benchmark $\mathrm{P} 1$ to $0.21 \mathrm{fb}$ in $\mathrm{P} 3$. In table 3 , we estimate the signal significance defined as $S / \sqrt{B}$ by keeping the mass gap between the gluino and the lightest neutralino fixed at $10 \mathrm{GeV}$ for the benchmark points.

We estimate that one can observe a signal at $5 \sigma$ significance at $13 \mathrm{TeV}$ with $30 \mathrm{fb}^{-1}$ luminosity up to a gluino mass of $1 \mathrm{TeV}$ while a gluino mass of $1.2 \mathrm{TeV}$ can be excluded at the same luminosity. At $100 \mathrm{fb}^{-1}$ luminosity the discovery reach goes up to $1.15 \mathrm{TeV}$, while we can exclude a gluino mass of up to $1.3 \mathrm{TeV}$. For the high luminosity Run of LHC $\left(3000 \mathrm{fb}^{-1}\right)$, we can discover a gluino mass up to $1.45 \mathrm{TeV}$, excluding about $1.55 \mathrm{TeV}$. $^{3}$

We would like to compare our results with the standard monojet strategy at $13 \mathrm{TeV}$. However, since this is not available yet, a rough estimate could be obtained by a rescaling of the cross section from the $8 \mathrm{TeV}$ result to the $13 \mathrm{TeV}$ by assuming that the acceptance times efficiency remains the same. This has already been performed by the authors of [28] for the high luminosity LHC Run $\left(3000 \mathrm{fb}^{-1}\right)$. They predict that one can exclude a gluino mass of $1.25 \mathrm{TeV}$ with a $\Delta M$ of $10 \mathrm{GeV}$, although in the context where $\operatorname{BR}\left(\tilde{g} \rightarrow q \bar{q} \tilde{\chi}_{1}^{0}\right)=100 \%$.

\footnotetext{
${ }^{3}$ For the large mass gap scenario, we easily obtain at least 4 jets from the gluino decay and additional QCD radiation jets. In this case the improved CMS and ATLAS multijet searches at $13 \mathrm{TeV}$ are expected to be quite sensitive (as we have already observed for the $8 \mathrm{TeV}$ LHC in section 3 ). Indeed we find that our search strategy, which targets a dijet + missing energy signature, suffers in this case, and we only manage to discover a gluino mass of $1.1 \mathrm{TeV}$ for a $\tilde{\chi}_{1}^{0}$ mass of $1 \mathrm{TeV}$ with $3000 \mathrm{fb}^{-1}$ luminosity.
} 


\begin{tabular}{|c|c|c|c|}
\hline & P1 & P2 & P3 \\
\hline$m_{\tilde{g}}, m_{\tilde{\chi}_{1}^{0}}(\mathrm{GeV})$ & 1005,999 & 1205,1195 & 1405,1395 \\
\hline$S / \sqrt{B}\left(30 \mathrm{fb}^{-1}\right)$ & 5.3 & 2.0 & 0.7 \\
\hline$S / \sqrt{B}\left(100 \mathrm{fb}^{-1}\right)$ & 9.7 & 3.7 & 1.27 \\
\hline$S / \sqrt{B}\left(3000 \mathrm{fb}^{-1}\right)$ & 53 & 20 & 7 \\
\hline
\end{tabular}

Table 3. The signal significances $(S / \sqrt{B})$ for different energies and luminosities for the benchmark points.

On this evidence the dijet + missing energy strategy devised here has a clear advantage, and we advocate the use of this strategy for $13 \mathrm{TeV}$ LHC.

\section{Conclusion}

We have performed an investigation of the MSSM region where the gluino-neutralino spectrum is compressed and the loop-induced decay $\tilde{g} \rightarrow g \tilde{\chi}_{1}^{0}$ is dominant over three-body decays. For small gluino-neutralino mass differences $\Delta M$, this decay mode is a sensitive probe of compressed spectra since it does not suffer from kinematical suppression factors. Within the scenario we investigated, namely with decoupled heavy scalars but with third generation squarks closer to the electroweak scale, the loop-induced decay of the gluino into a pure higgsino-like neutralino dominates over other branching fractions until the $t b$ threshold is crossed, when $\Delta M$ increases. This occurs whatever the hierarchy between the stops and the sbottoms, albeit larger branching fractions are reached when $m_{\widetilde{b}_{1}} \gg m_{\widetilde{t}_{1}}$. On the contrary, the dominance of the radiative gluino decay into a bino-like neutralino or mixed binohiggsino one over the other decay modes strongly depends on this hierarchy and other threebody decay modes start to be significant before the opening of the $t b$ kinematical threshold.

Adopting a Simplified Model approach, where the gluino and the neutralino $\tilde{\chi}_{1}^{0}$ are the only relevant superparticles accessible at the $\operatorname{LHC}$, and $\operatorname{BR}\left(\tilde{g} \rightarrow g \tilde{\chi}_{1}^{0}\right)=100 \%$, we reinterpreted some ATLAS and CMS $8 \mathrm{TeV}$ SUSY searches to derive $95 \%$ CL exclusion contours in the $m_{\tilde{g}}-m_{\tilde{\chi}_{1}^{0}}$ plane. This decay process is particularly interesting to probe small $\Delta M$. The reinterpretation of the ATLAS and CMS analyses has been performed through the MadAnalysis5 framework [80, 81] and publicly available on its Public Analysis Database web page [92]. The recasted monojet analysis enables us to illustrate that, had it been used by the experimental collaborations to constrain the loop-induced gluino decay, this search is sensitive to degenerate gluino-LSP scenarios. In turn, degenerate situations with gluino masses up to $m_{\tilde{g}} \simeq 750 \mathrm{GeV}$ could have been excluded. This surpasses the official maximal value $m_{\tilde{g}} \simeq 600 \mathrm{GeV}$ reached by the experimental collaborations using the process $\tilde{g} \rightarrow q \bar{q} \tilde{\chi}_{1}^{0}$.

Then, concentrating on a pure di-jet search, we designed a dedicated search strategy to discover the gluino through the $\tilde{g} \rightarrow g \tilde{\chi}_{1}^{0}$ channel. For a mass gap fixed at $\Delta M=10 \mathrm{GeV}$, a signal at $5 \sigma$ significance at $13 \mathrm{TeV}$ with $30 \mathrm{fb}^{-1}$ luminosity could be observed up to a gluino mass $m_{\tilde{g}}=1 \mathrm{TeV}$, while the discovery reach goes up to $1.2 \mathrm{TeV}$. At $100 \mathrm{fb}^{-1}$ luminosity the 
discovery reach goes up to $1.15 \mathrm{TeV}$, while we can exclude up to $1.3 \mathrm{TeV}$. A high-luminosity LHC could discover a gluino mass up to $1.45 \mathrm{TeV}$ while excluding about $1.55 \mathrm{TeV}$.

In addition, our Monte-Carlo investigation indicated an interesting point. Indeed, a pure di-jet search could be as sensitive as a monojet search and maybe even better. The underlying reason is related to the fact that a heavy gluino radiates a lot of high- $p_{T}$ ISR jets, even for small $\Delta M$. Of course this has to be taken with a grain of salt since our investigation was performed only a the Monte-Carlo level and only a full detector simulation could give a clear cut answer, but this result is nevertheless worth to be pointed out.

The discovery of a $125 \mathrm{GeV}$ Higgs boson suggests that the scalar superpartners may lie in the multi-TeV range. Direct search of such heavy particles is impossible at the LHC. If the scalar mass scale $\tilde{m}$ is much less than $\tilde{m} \lesssim \mathcal{O}\left(10^{3}\right) \mathrm{TeV}$, the gluino two-body decay is quite important in probing indirectly $\tilde{m}$. In the end, it may well be our only probe at our disposal in the case of SUSY scenarios with heavy scalars and compressed SUSY, if the gluino decays promptly in the detector. We hope our work will motivate the experimental collaborations to perform a dedicated analysis to search for this decay in order to make the most of the LHC potential.

\section{Acknowledgments}

We thank our experimental colleagues Jamie Boyd, Tommaso Lari and Jalal Abdallah for additional information on the ATLAS analyses. We appreciated greatly the help of Sabine Kraml for her comments and suggestions on the manuscript and of Bryan Zaldivar concerning the monojet analysis. GC would also like to thank Mathieu Aurousseau for the help with ROOT. The work of GC is supported by the Theory-LHC-France initiative of CNRS/IN2P3. The work of DS is supported by the French ANR, project DMAstroLHC, ANR-12-BS05-0006, and by the Investissements d'avenir, Labex ENIGMASS.

\section{A Cutflow for the ATLAS monojet analysis}

We give below the cut flow table for the comparison between the ATLAS monojet analysis and the recasted MA5 one. The monojets analysis targets the flavour-violating decay $\tilde{t} \rightarrow c \tilde{\chi}_{1}^{0}$ with a branching fraction of $100 \%$ using a monojet and c-tagged strategies. We only recasted the monojet strategy. The dataset corresponds to $20.3 \mathrm{fb}^{-1}$ of integrated luminosity at $\sqrt{\mathrm{s}}=8 \mathrm{TeV}$.

\section{B Cutflow for the ATLAS multi-jet analysis}

This ATLAS analysis [86] searches for new physics in the 0 lepton + multi-jets (2-6 jets) + missing energy $\left(E_{T}^{\text {miss }}\right)$ final state. The dataset corresponds to $20.3 \mathrm{fb}^{-1}$ of integrated luminosity at $\sqrt{\mathrm{s}}=8 \mathrm{TeV}$. In the context of supersymmetry, the analysis targets gluino pair production $(\tilde{g} \tilde{g})$, squark pair production $(\tilde{q} \tilde{q})$, and squark gluino production $(\tilde{q} \tilde{g})$. For each of the production modes the following cases are investigated; 


\begin{tabular}{|c|c|c|c|c|c|c|}
\hline \multirow{2}{*}{$\begin{array}{l}\operatorname{Benchmark}\left(m_{\tilde{g}}, m_{\tilde{\chi}_{1}^{0}}\right) \\
\text { cut }\end{array}$} & \multicolumn{2}{|c|}{$(200 / 125) \mathrm{GeV}$} & \multicolumn{2}{|c|}{$(200 / 195) \mathrm{GeV}$} & \multicolumn{2}{|c|}{$(250 / 245) \mathrm{GeV}$} \\
\hline & $\begin{array}{l}\text { MA } 5 \\
\text { result }\end{array}$ & $\begin{array}{c}\text { CMS } \\
\text { result }\end{array}$ & $\begin{array}{l}\text { MA } 5 \\
\text { result }\end{array}$ & $\begin{array}{l}\text { CMS } \\
\text { result }\end{array}$ & $\begin{array}{l}\text { MA } 5 \\
\text { result }\end{array}$ & $\begin{array}{l}\text { CMS } \\
\text { result }\end{array}$ \\
\hline$E_{T}^{\text {miss }}>80 \mathrm{GeV}$ Filter & 192812.8 & 181902.0 & 104577.6 & 103191.0 & 36055.4 & 48103.0 \\
\hline$E_{T}^{\text {miss }}>100 \mathrm{GeV}$ & 136257.1 & 97217.0 & 82619.0 & 64652.0 & 29096.3 & 23416.0 \\
\hline Event cleaning & - & 82131.0 & - & 57566.0 & - & 21023.0 \\
\hline Lepton veto & 134894.2 & 81855.0 & 82493.9 & 57455.0 & 29041.8 & 20986.0 \\
\hline$N_{\text {jets }} \leq 3$ & 101653.7 & 59315.0 & 75391.5 & 52491.0 & 26295.2 & 18985.0 \\
\hline$\Delta \phi\left(E_{T}^{\text {miss }}\right.$, jets $)>0.4$ & 95568.8 & 54295.0 & 70888.1 & 49216.0 & 24676.9 & 17843.0 \\
\hline$p_{T}\left(j_{1}\right)>150 \mathrm{GeV}$ & 17282.8 & 14220.0 & 25552.0 & 20910.0 & 9652.1 & 8183.0 \\
\hline$E_{T}^{\text {miss }}>150 \mathrm{GeV}$ & 10987.8 & 9468.0 & 21569.1 & 18297.0 & 8363.0 & 7290.0 \\
\hline \multicolumn{7}{|c|}{ M1 Signal Region } \\
\hline$p_{T}\left(j_{1}\right)>280 \mathrm{GeV}$ & 2031.2 & 1627.0 & 4922.0 & 3854.0 & 2156.1 & 1748.0 \\
\hline$E_{T}^{\text {miss }}>220 \mathrm{GeV}$ & 1517.6 & 1276.0 & 4628.4 & 3722.0 & 2022.9 & 1694.0 \\
\hline \multicolumn{7}{|c|}{ M2 Signal Region } \\
\hline$p_{T}\left(j_{1}\right)>340 \mathrm{GeV}$ & 858.0 & 721.0 & 2509.0 & 1897.0 & 1107.4 & 882.0 \\
\hline$E_{T}^{\text {miss }}>340 \mathrm{GeV}$ & 344.4 & 282.0 & 1758.9 & 1518.0 & 817.5 & 736.0 \\
\hline \multicolumn{7}{|c|}{ M3 Signal Region } \\
\hline$p_{T}\left(j_{1}\right)>450 \mathrm{GeV}$ & 204.3 & 169.0 & 773.3 & 527.0 & 376.1 & 279.0 \\
\hline$E_{T}^{\text {miss }}>450 \mathrm{GeV}$ & 61.3 & 64.0 & 476.8 & 415.0 & 268.0 & 230.0 \\
\hline
\end{tabular}

Table 4. Cutflow comparison for three benchmark point of the monojet analysis targeting $\tilde{t} \rightarrow c \tilde{\chi}_{1}^{0}$ in [85]. The official ATLAS numbers are taken from [113].

- Gluino pair production:

1. Direct: $\tilde{g} \rightarrow q \bar{q} \tilde{\chi}_{1}^{0}$.

2. One step: $\tilde{g} \rightarrow q q^{\prime} \tilde{\chi}_{1}^{ \pm}$followed by $\tilde{\chi}_{1}^{ \pm} \rightarrow W \tilde{\chi}_{1}^{0}$

- Squark pair production:

1. Direct: $\tilde{q} \rightarrow q \tilde{\chi}_{1}^{0}$.

2. One step: $\tilde{q} \rightarrow q^{\prime} \tilde{\chi}_{1}^{ \pm}$followed by $\tilde{\chi}_{1}^{ \pm} \rightarrow W \tilde{\chi}_{1}^{0}$

- Squark gluino production:

1. Direct: $\tilde{g} \rightarrow q \bar{q} \tilde{\chi}_{1}^{0}, \tilde{q} \rightarrow q \tilde{\chi}_{1}^{0}$.

For all of the above cases, all branching ratios are assumed to be $100 \%$ and the rest of the spectrum is decoupled from this set. 


\begin{tabular}{|l|l|l|l|l|l|l|}
\hline decay to LSP & $\begin{array}{l}2 \mathrm{jm} \\
(\tilde{q} \tilde{q})\end{array}$ & $\begin{array}{l}2 \mathrm{jt} \\
(\tilde{q} \tilde{q})\end{array}$ & $\begin{array}{l}3 \mathrm{j} \\
(\tilde{g} \tilde{q})\end{array}$ & $\begin{array}{l}4 \mathrm{jl}- \\
(\tilde{q} \tilde{q})\end{array}$ & $\begin{array}{l}4 \mathrm{jl} \\
(\tilde{g} \tilde{g})\end{array}$ & $\begin{array}{l}4 \mathrm{jt} \\
(\tilde{g} \tilde{g})\end{array}$ \\
\hline & direct & direct & direct & direct & direct & direct \\
\hline$m_{\tilde{q} / \tilde{g}}$ & 475 & 1000 & 1612 & 400 & 800 & 1425 \\
\hline$m_{\tilde{\chi}_{1}^{ \pm}}$ & - & - & - & - & - & - \\
\hline$m_{\tilde{\chi}_{1}^{0}}$ & 425 & 100 & 337 & 250 & 650 & 75 \\
\hline
\end{tabular}

Table 5. The benchmark scenarios used for the valiaion of the 10 signal regions. For the direct squark pair or gluino pair production, the rest of the spectrum is decoupled. For squark-gluino production, the gluino and squark masses are assumed to be degenerate. All masses are in GeV. The SLHA files were obtained from http://hepdata.cedar.ac.uk/view/ins1298722.

\begin{tabular}{|l|l|l|l|l|l|l|}
\hline & $2 \mathrm{jm}$ & $2 \mathrm{jt}$ & $3 \mathrm{j}$ & $4 \mathrm{jl}-$ & $4 \mathrm{jl}$ & $4 \mathrm{jt}$ \\
\hline$E_{T}^{\text {miss }} / \sqrt{H_{T}}$ & 15 & 15 & - & 10 & 10 & - \\
\hline$E_{T}^{\text {miss }} / M_{\text {eff }}\left(N_{j}\right)$ & - & - & 0.3 & - & - & - \\
\hline$M_{\text {eff }}$ (incl) & 1200 & 1600 & 2200 & 700 & 1000 & 2200 \\
\hline
\end{tabular}

Table 6. The signal region specific cuts. All energy units are in GeV.

\begin{tabular}{|l|c|c|c|c|c|c|}
\hline Benchmark/SR & \multicolumn{2}{|c|}{$2 \mathrm{jm}(\tilde{q} \tilde{q})$} & \multicolumn{2}{c|}{$2 \mathrm{jt}(\tilde{q} \tilde{q})$} & \multicolumn{2}{c|}{$3 \mathrm{j}(\tilde{g} \tilde{q})$} \\
cut & $\begin{array}{c}\text { MA 5 } \\
\text { result }\end{array}$ & $\begin{array}{c}\text { ATLAS } \\
\text { result }\end{array}$ & $\begin{array}{c}\text { MA 5 } \\
\text { result }\end{array}$ & $\begin{array}{c}\text { ATLAS } \\
\text { result }\end{array}$ & $\begin{array}{c}\text { MA 5 } \\
\text { result }\end{array}$ & $\begin{array}{c}\text { ATLAS } \\
\text { result }\end{array}$ \\
\hline $\begin{array}{l}E_{T}^{\text {miss }}>160, \\
p_{T}\left(j_{1}, j_{2}\right)>130,60\end{array}$ & 1656.1 & 1781.2 & 62.1 & 61.6 & 18.8 & 18.6 \\
\hline$p_{T}\left(j_{3}\right)>60$ & - & - & - & - & 15.1 & 14.8 \\
\hline$\Delta \phi\left(j_{i}, E_{T}^{\text {miss }}\right)>0.4$ & 1295.9 & 1462.7 & 56.9 & 55.7 & 13.3 & 12.9 \\
\hline$E_{T}^{\text {miss }} / \sqrt{H_{T}}$ & 449.1 & 566.1 & 40.1 & 38.5 & - & - \\
\hline$E_{T}^{\text {miss }} / M_{\text {eff }}\left(N_{j}\right)$ & - & - & - & - & 10.1 & 9.6 \\
\hline$M_{\text {eff }}(\mathrm{incl})$ & 122.2 & 102.4 & 23.8 & 21.7 & 6.2 & 5.9 \\
\hline
\end{tabular}

Table 7. Cutflows for signal regions $2 \mathrm{jm}, 2 \mathrm{jt}$ and $3 \mathrm{j}$, compared to the official ATLAS results documented in [86]. All energy units are in GeV.

In the following tables we summarize the benchmark points used (table 5), the cuts used for different signal regions (up to 4 jets, table 6), and finally the comparison between the ATLAS multi-jet analysis and the recasted MA5 one (tables 7-8). Further details about the validation can be found on the web page [92]. 


\begin{tabular}{|l|c|c|c|c|c|c|}
\hline Benchmark/SR & \multicolumn{2}{|c|}{$4 \mathrm{jl}-(\tilde{q} \tilde{q})$} & \multicolumn{2}{c|}{$4 \mathrm{jl}(\tilde{g} \tilde{g})$} & \multicolumn{2}{c|}{$4 \mathrm{jt}(\tilde{g} \tilde{g})$} \\
cut & $\begin{array}{c}\text { ATLAS } \\
\text { result }\end{array}$ & $\begin{array}{c}\text { MA 5 } \\
\text { result }\end{array}$ & $\begin{array}{c}\text { ATLAS } \\
\text { result }\end{array}$ & $\begin{array}{c}\text { MA 5 } \\
\text { result }\end{array}$ & $\begin{array}{c}\text { ATLAS } \\
\text { result }\end{array}$ & $\begin{array}{c}\text { MA 5 } \\
\text { result }\end{array}$ \\
\hline $\begin{array}{l}E_{T}^{\text {miss }}>160, \\
p_{T}\left(j_{1}, j_{2}\right)>130,60\end{array}$ & 16135.8 & 15097 & 634.6 & 679.0 & 13.2 & 12.7 \\
\hline$p_{T}\left(j_{3}, j_{4}\right)>60$ & 2331 & 2112 & 211.4 & 185.7 & 12.0 & 12.0 \\
\hline $\begin{array}{l}\Delta \phi\left(j_{i} \leq 3\right. \\
\left.E_{T}^{\text {miss }}\right)>0.4\end{array}$ & 1813.7 & 1723.0 & 154.6 & 144.9 & 8.4 & 8.9 \\
$\Delta \phi\left(j_{4}, E_{T}^{\text {miss }}\right)>0.2$ & & & & & & \\
\hline$E_{T}^{\text {miss }} / \sqrt{H_{T}}$ & 1009 & 943 & 98.7 & 84.4 & - & - \\
\hline$E_{T}^{\text {miss }} / M_{\text {eff }}\left(N_{j}\right)$ & - & - & - & - & 4.8 & 5.5 \\
\hline $\mathrm{M}_{\text {eff }}($ incl $)$ & 884 & 843 & 39.5 & 41.5 & 2.5 & 2.9 \\
\hline
\end{tabular}

Table 8. Cutflows for signal regions 4jl-,4jl and 4jt, compared to the official ATLAS results documented in [86].

Open Access. This article is distributed under the terms of the Creative Commons Attribution License (CC-BY 4.0), which permits any use, distribution and reproduction in any medium, provided the original author(s) and source are credited.

\section{References}

[1] ATLAS supersymmetry (SUSY) searches webpage, https://twiki.cern.ch/twiki/bin/view/AtlasPublic/SupersymmetryPublicResults.

[2] CMS supersymmetry physics results webpage, https://twiki.cern.ch/twiki/bin/view/CMSPublic/PhysicsResultsSUS.

[3] ATLAS exotics public results webpage, https://twiki.cern.ch/twiki/bin/view/AtlasPublic/ExoticsPublicResults.

[4] CMS exotica public physics results webpage, https://twiki.cern.ch/twiki/bin/view/CMSPublic/PhysicsResultsEXO.

[5] ATLAS and CMS collaborations, Combined measurement of the Higgs boson mass in pp collisions at $\sqrt{s}=7$ and $8 \mathrm{TeV}$ with the ATLAS and CMS experiments, Phys. Rev. Lett. 114 (2015) 191803 [arXiv: 1503.07589] [INSPIRE].

[6] ATLAS collaboration, Summary of the searches for squarks and gluinos using $\sqrt{s}=8 \mathrm{TeV}$ pp collisions with the ATLAS experiment at the LHC, JHEP 10 (2015) 054 [arXiv: 1507.05525] [INSPIRE].

[7] ATLAS and CMS collaborations, Supersymmetry searches at the LHC, in Proceedings, $32^{\text {nd }}$ International Symposium on Physics in Collision (PIC 2012), (2012), pg. 241 [arXiv: 1211.3887] [INSPIRE].

[8] ATLAS collaboration, H. Okawa, Interpretations of SUSY searches in ATLAS with simplified models, in Particles and fields. Proceedings, Meeting of the Division of the American Physical Society, DPF 2011, Providence U.S.A. August 9-13 2011 [arXiv: 1110.0282] [INSPIRE]. 
[9] CMS collaboration, Interpretation of searches for supersymmetry with simplified models, Phys. Rev. D 88 (2013) 052017 [arXiv:1301.2175] [INSPIRE].

[10] J. Alwall, P. Schuster and N. Toro, Simplified models for a first characterization of new physics at the LHC, Phys. Rev. D 79 (2009) 075020 [arXiv:0810.3921] [INSPIRE].

[11] D.S.M. Alves, E. Izaguirre and J.G. Wacker, Where the sidewalk ends: jets and missing energy search strategies for the 7 TeV LHC, JHEP 10 (2011) 012 [arXiv:1102.5338] [INSPIRE].

[12] LHC New Physics Working Group collaboration, D. Alves, Simplified models for LHC new physics searches, J. Phys. G 39 (2012) 105005 [arXiv:1105.2838] [InSPIRE].

[13] H.K. Dreiner, M. Krämer and J. Tattersall, How low can SUSY go? Matching, monojets and compressed spectra, Europhys. Lett. 99 (2012) 61001 [arXiv:1207.1613] [INSPIRE].

[14] J. Alwall, M.-P. Le, M. Lisanti and J.G. Wacker, Searching for directly decaying gluinos at the Tevatron, Phys. Lett. B 666 (2008) 34 [arXiv:0803.0019] [INSPIRE].

[15] E. Izaguirre, M. Manhart and J.G. Wacker, Bigger, better, faster, more at the LHC, JHEP 12 (2010) 030 [arXiv: 1003.3886] [INSPIRE].

[16] J. Alwall, M.-P. Le, M. Lisanti and J.G. Wacker, Model-independent jets plus missing energy searches, Phys. Rev. D 79 (2009) 015005 [arXiv:0809.3264] [InSPIRE].

[17] T.J. LeCompte and S.P. Martin, Large Hadron Collider reach for supersymmetric models with compressed mass spectra, Phys. Rev. D 84 (2011) 015004 [arXiv:1105.4304] [INSPIRE].

[18] T.J. LeCompte and S.P. Martin, Compressed supersymmetry after $1 \mathrm{fb}^{-1}$ at the Large Hadron Collider, Phys. Rev. D 85 (2012) 035023 [arXiv:1111.6897] [InSPIRE].

[19] B. Bhattacherjee and K. Ghosh, Degenerate SUSY search at the 8 Te V LHC, arXiv: 1207.6289 [INSPIRE].

[20] ATLAS collaboration, Search for strong production of supersymmetric particles in final states with missing transverse momentum and at least three b-jets at $\sqrt{s}=8 \mathrm{TeV}$ proton-proton collisions with the ATLAS detector, JHEP 10 (2014) 024 [arXiv:1407.0600] [INSPIRE].

[21] ATLAS collaboration, Search for new phenomena in final states with large jet multiplicities and missing transverse momentum at $\sqrt{s}=8 \mathrm{TeV}$ proton-proton collisions using the ATLAS experiment, JHEP 10 (2013) 130 [Erratum ibid. 01 (2014) 109] [arXiv:1308.1841] [INSPIRE].

[22] CMS collaboration, Search for supersymmetry in pp collisions at $\sqrt{s}=8$ TeV in events with a single lepton, large jet multiplicity and multiple b jets, Phys. Lett. B 733 (2014) 328 [arXiv:1311.4937] [INSPIRE].

[23] CMS collaboration, Search for new physics in events with same-sign dileptons and jets in pp collisions at $\sqrt{s}=8 \mathrm{TeV}$, JHEP 01 (2014) 163 [Erratum ibid. 01 (2015) 014] [arXiv:1311.6736] [INSPIRE].

[24] CMS collaboration, Exclusion limits on gluino and top-squark pair production in natural SUSY scenarios with inclusive razor and exclusive single-lepton searches at $8 \mathrm{TeV}$., CMS-PAS-SUS-14-011, CERN, Geneva Switzerland (2014).

[25] M. Carena, A. Freitas and C.E.M. Wagner, Light stop searches at the LHC in events with one hard photon or jet and missing energy, JHEP 10 (2008) 109 [arXiv:0808.2298] [INSPIRE]. 
[26] E. Alvarez and Y. Bai, Reach the bottom line of the sbottom search, JHEP 08 (2012) 003 [arXiv:1204.5182] [INSPIRE].

[27] H. Dreiner, M. Krämer and J. Tattersall, Exploring QCD uncertainties when setting limits on compressed supersymmetric spectra, Phys. Rev. D 87 (2013) 035006 [arXiv:1211.4981] [INSPIRE].

[28] A. Arbey, M. Battaglia and F. Mahmoudi, Monojet searches for MSSM simplified models, arXiv: 1506.02148 [INSPIRE].

[29] A. Chatterjee, A. Choudhury, A. Datta and B. Mukhopadhyaya, Gluino mass limits with sbottom NLSP in coannihilation scenarios, JHEP 01 (2015) 154 [arXiv:1411.6467] [INSPIRE].

[30] B. He, T. Li and Q. Shafi, Impact of LHC searches on NLSP top squark and gluino mass, JHEP 05 (2012) 148 [arXiv:1112.4461] [INSPIRE].

[31] M. Drees, M. Hanussek and J.S. Kim, Light stop searches at the LHC with monojet events, Phys. Rev. D 86 (2012) 035024 [arXiv:1201.5714] [INSPIRE].

[32] ATLAS collaboration, ATLAS Run 1 searches for direct pair production of third-generation squarks at the Large Hadron Collider, Eur. Phys. J. C 75 (2015) 510 [arXiv:1506.08616] [INSPIRE].

[33] CMS collaboration, Search for top squarks decaying to a charm quark and a neutralino in events with a jet and missing transverse momentum, CMS-PAS-SUS-13-009, CERN, Geneva Switzerland (2013).

[34] G. Ferretti, R. Franceschini, C. Petersson and R. Torre, Light stop squarks and b-tagging, in $14^{\text {th }}$ Hellenic School and Workshops on Elementary Particle Physics and Gravity, Corfu Attiki Greece September 3-21 2014 [PoS (CORFU2014) 076] [arXiv : 1506. 00604] [INSPIRE].

[35] M. Toharia and J.D. Wells, Gluino decays with heavier scalar superpartners, JHEP 02 (2006) 015 [hep-ph/0503175] [InSPIRE].

[36] E. Ma and G.-G. Wong, Two-body radiative gluino decays, Mod. Phys. Lett. A 3 (1988) 1561 [INSPIRE].

[37] R. Barbieri, G. Gamberini, G.F. Giudice and G. Ridolfi, Constraining supergravity models from gluino production, Nucl. Phys. B 301 (1988) 15 [INSPIRE].

[38] H.E. Haber and G.L. Kane, Gluino decays and experimental signatures, Nucl. Phys. B 232 (1984) 333 [INSPIRE].

[39] H. Baer, X. Tata and J. Woodside, Phenomenology of gluino decays via loops and top quark Yukawa coupling, Phys. Rev. D 42 (1990) 1568 [InSPIRE].

[40] A. Bartl, W. Majerotto, B. Mosslacher, N. Oshimo and S. Stippel, Gluino and squark decays into heavy top quarks, Phys. Rev. D 43 (1991) 2214 [InSPIRE].

[41] P. Gambino, G.F. Giudice and P. Slavich, Gluino decays in split supersymmetry, Nucl. Phys. B 726 (2005) 35 [hep-ph/0506214] [INSPIRE].

[42] R. Sato, S. Shirai and K. Tobioka, Gluino decay as a probe of high scale supersymmetry breaking, JHEP 11 (2012) 041 [arXiv:1207.3608] [INSPIRE].

[43] J.D. Wells, Implications of supersymmetry breaking with a little hierarchy between gauginos and scalars, in $11^{\text {th }}$ International Conference on Supersymmetry and the Unification of Fundamental Interactions (SUSY 2003), Tucson AZ U.S.A. June 5-10 2003 [hep-ph/0306127] [INSPIRE]. 
[44] J.D. Wells, PeV-scale supersymmetry, Phys. Rev. D 71 (2005) 015013 [hep-ph/0411041] [INSPIRE].

[45] N. Arkani-Hamed, S. Dimopoulos, G.F. Giudice and A. Romanino, Aspects of split supersymmetry, Nucl. Phys. B 709 (2005) 3 [hep-ph/0409232] [INSPIRE].

[46] G.F. Giudice and A. Romanino, Split supersymmetry, Nucl. Phys. B 699 (2004) 65 [Erratum ibid. B 706 (2005) 65] [hep-ph/0406088] [INSPIRE].

[47] N. Arkani-Hamed and S. Dimopoulos, Supersymmetric unification without low energy supersymmetry and signatures for fine-tuning at the LHC, JHEP 06 (2005) 073 [hep-th/0405159] [INSPIRE].

[48] A. Arvanitaki, N. Craig, S. Dimopoulos and G. Villadoro, Mini-split, JHEP 02 (2013) 126 [arXiv:1210.0555] [INSPIRE].

[49] K. Harigaya, M. Ibe and T.T. Yanagida, A closer look at gaugino masses in pure gravity mediation model/minimal split SUSY model, JHEP 12 (2013) 016 [arXiv:1310.0643] [INSPIRE].

[50] L.J. Hall and Y. Nomura, Spread supersymmetry, JHEP 01 (2012) 082 [arXiv:1111.4519] [INSPIRE].

[51] M. Ibe and T.T. Yanagida, The lightest Higgs boson mass in pure gravity mediation model, Phys. Lett. B 709 (2012) 374 [arXiv:1112.2462] [INSPIRE].

[52] M. Ibe, S. Matsumoto and T.T. Yanagida, Pure gravity mediation with $m_{3 / 2}=10-100 \mathrm{TeV}$, Phys. Rev. D 85 (2012) 095011 [arXiv:1202.2253] [InSPIRE].

[53] J.L. Evans and K.A. Olive, Universality in pure gravity mediation with vector multiplets, Phys. Rev. D 90 (2014) 115020 [arXiv:1408.5102] [INSPIRE].

[54] ATLAS collaboration, Search for long-lived stopped R-hadrons decaying out-of-time with pp collisions using the ATLAS detector, Phys. Rev. D 88 (2013) 112003 [arXiv:1310.6584] [INSPIRE].

[55] ATLAS collaboration, Searches for heavy long-lived charged particles with the ATLAS detector in proton-proton collisions at $\sqrt{s}=8 \mathrm{TeV}$, JHEP 01 (2015) 068 [arXiv:1411.6795] [INSPIRE].

[56] D. Feldman, Z. Liu and P. Nath, Light Higgses at the Tevatron and at the LHC and observable dark matter in SUGRA and D-branes, Phys. Lett. B 662 (2008) 190 [arXiv: 0711.4591] [INSPIRE].

[57] D. Feldman, Z. Liu and P. Nath, Sparticles at the LHC, JHEP 04 (2008) 054 [arXiv:0802.4085] [INSPIRE].

[58] D. Feldman, Z. Liu and P. Nath, Gluino NLSP, dark matter via gluino coannihilation and LHC signatures, Phys. Rev. D 80 (2009) 015007 [arXiv:0905.1148] [InSPIRE].

[59] N. Chen, D. Feldman, Z. Liu, P. Nath and G. Peim, Low mass gluino within the sparticle landscape, implications for dark matter and early discovery prospects at LHC-7, Phys. Rev. D 83 (2011) 035005 [arXiv: 1011.1246] [INSPIRE].

[60] H. Baer, A. Mustafayev, E.-K. Park, S. Profumo and X. Tata, Mixed Higgsino dark matter from a reduced $\mathrm{SU}(3)$ gaugino mass: consequences for dark matter and collider searches, JHEP 04 (2006) 041 [hep-ph/0603197] [INSPIRE]. 
[61] H. Baer, A. Mustafayev, S. Profumo and X. Tata, Probing SUSY beyond the reach of LEP2 at the Fermilab Tevatron: low $\left|M^{3}\right|$ dark matter models, Phys. Rev. D 75 (2007) 035004 [hep-ph/0610154] [INSPIRE].

[62] H. Baer, A. Mustafayev, E.-K. Park and X. Tata, Collider signals and neutralino dark matter detection in relic-density-consistent models without universality, JHEP 05 (2008) 058 [arXiv:0802.3384] [INSPIRE].

[63] M. Guchait, D.P. Roy and D. Sengupta, Probing a mixed neutralino dark matter model at the 7 TeV LHC, Phys. Rev. D 85 (2012) 035024 [arXiv:1109.6529] [INSPIRE].

[64] I. Gogoladze, R. Khalid and Q. Shafi, Yukawa unification and neutralino dark matter in $\mathrm{SU}(4)_{c} \times \mathrm{SU}(2)_{L} \times \mathrm{SU}(2)_{R}$, Phys. Rev. D $79(2009) 115004$ [arXiv:0903.5204] [inSPIRE].

[65] I. Gogoladze, R. Khalid and Q. Shafi, Coannihilation scenarios and particle spectroscopy in $\mathrm{SU}(4)_{c} \times \mathrm{SU}(2)_{L} \times \mathrm{SU}(2)_{R}$, Phys. Rev. D 80 (2009) 095016 [arXiv:0908.0731] [InSPIRE].

[66] M. Adeel Ajaib, T. Li, Q. Shafi and K. Wang, NLSP gluino search at the Tevatron and early LHC, JHEP 01 (2011) 028 [arXiv:1011.5518] [INSPIRE].

[67] S. Raza, Q. Shafi and C.S. Ün, NLSP gluino and NLSP stop scenarios from b- $\tau$ Yukawa unification, Phys. Rev. D 92 (2015) 055010 [arXiv:1412.7672] [INSPIRE].

[68] P. Grajek, A. Mariotti and D. Redigolo, Phenomenology of general gauge mediation in light of a $125 \mathrm{GeV}$ Higgs, JHEP 07 (2013) 109 [arXiv: 1303.0870] [INSPIRE].

[69] D.A. Dicus, S. Nandi and J. Woodside, Collider signals of a superlight gravitino, Phys. Rev. D 41 (1990) 2347 [INSPIRE].

[70] M. Drees and J. Woodside, Signals for a superlight gravitino at the LHC, in ECFA Large Hadron Collider (LHC) Workshop: physics and Instrumentation, http://alice.cern.ch/format/showfull?sysnb=0128115, Aachen Germany October 4-9 1990.

[71] D.A. Dicus and S. Nandi, New collider bound on light gravitino mass, Phys. Rev. D 56 (1997) 4166 [hep-ph/9611312] [INSPIRE].

[72] J. Kim, J.L. Lopez, D.V. Nanopoulos, R. Rangarajan and A. Zichichi, Light gravitino production at hadron colliders, Phys. Rev. D 57 (1998) 373 [hep-ph/9707331] [INSPIRE].

[73] A. Brignole, F. Feruglio, M.L. Mangano and F. Zwirner, Signals of a superlight gravitino at hadron colliders when the other superparticles are heavy, Nucl. Phys. B 526 (1998) 136 [Erratum ibid. B 582 (2000) 759] [hep-ph/9801329] [INSPIRE].

[74] K.S. Babu, T. Enkhbat and B. Mukhopadhyaya, Split supersymmetry from anomalous U(1), Nucl. Phys. B 720 (2005) 47 [hep-ph/0501079] [InSPIRE].

[75] M. Klasen and G. Pignol, New results for light gravitinos at hadron colliders: Tevatron limits and LHC perspectives, Phys. Rev. D 75 (2007) 115003 [hep-ph/0610160] [InSPIRE].

[76] P. de Aquino, F. Maltoni, K. Mawatari and B. Oexl, Light gravitino production in association with gluinos at the LHC, JHEP 10 (2012) 008 [arXiv:1206.7098] [INSPIRE].

[77] ATLAS collaboration, Searches for heavy long-lived sleptons and R-hadrons with the ATLAS detector in pp collisions at $\sqrt{s}=7$ TeV, Phys. Lett. B 720 (2013) 277 [arXiv: 1211.1597] [INSPIRE].

[78] CMS collaboration, Searches for long-lived charged particles in pp collisions at $\sqrt{s}=7$ and 8 TeV, JHEP 07 (2013) 122 [arXiv:1305. 0491] [INSPIRE].

[79] ATLAS collaboration, Limits on metastable gluinos from ATLAS SUSY searches at $8 \mathrm{TeV}$, ATLAS-CONF-2014-037, CERN, Geneva Switzerland (2014). 
[80] E. Conte, B. Fuks and G. Serret, MadAnalysis 5, a user-friendly framework for collider phenomenology, Comput. Phys. Commun. 184 (2013) 222 [arXiv:1206.1599] [INSPIRE].

[81] E. Conte, B. Dumont, B. Fuks and C. Wymant, Designing and recasting LHC analyses with MadAnalysis 5, Eur. Phys. J. C 74 (2014) 3103 [arXiv:1405.3982] [INSPIRE].

[82] B. Dumont, B. Fuks, S. Kraml, S. Bein, G. Chalons, E. Conte et al., Toward a public analysis database for LHC new physics searches using MadAnalysis 5, Eur. Phys. J. C 75 (2015) 56 [arXiv: 1407.3278] [INSPIRE].

[83] A. Djouadi, M.M. Muhlleitner and M. Spira, Decays of supersymmetric particles: the program SUSY-HIT (SUspect-SdecaY-HDECAY-InTerface), Acta Phys. Polon. B 38 (2007) 635 [hep-ph/0609292] [INSPIRE].

[84] J. Ellis, F. Luo and K.A. Olive, Gluino coannihilation revisited, JHEP 09 (2015) 127 [arXiv: 1503.07142] [INSPIRE].

[85] ATLAS collaboration, Search for pair-produced third-generation squarks decaying via charm quarks or in compressed supersymmetric scenarios in pp collisions at $\sqrt{s}=8 \mathrm{TeV}$ with the ATLAS detector, Phys. Rev. D 90 (2014) 052008 [arXiv:1407.0608] [INSPIRE].

[86] ATLAS collaboration, Search for squarks and gluinos with the ATLAS detector in final states with jets and missing transverse momentum using $\sqrt{s}=8$ TeV proton-proton collision data, JHEP 09 (2014) 176 [arXiv:1405.7875] [INSPIRE].

[87] CMS collaboration, Search for new physics in the multijet and missing transverse momentum final state in proton-proton collisions at $\sqrt{s}=8 \mathrm{TeV}$, JHEP 06 (2014) 055 [arXiv: 1402.4770] [INSPIRE].

[88] S. Bein and D. Sengupta, MadAnalysis 5 implementation of CMS-SUS-13-012, (2014) [INSPIRE].

[89] ATLAS collaboration, Search for squarks and gluinos with the ATLAS detector in final states with jets and missing transverse momentum using $\sqrt{s}=8 \mathrm{TeV}$ proton-proton collision data, JHEP 09 (2014) 176 [arXiv:1405.7875] [INSPIRE].

[90] ATLAS collaboration, Search for pair-produced third-generation squarks decaying via charm quarks or in compressed supersymmetric scenarios in pp collisions at $\sqrt{s}=8 \mathrm{TeV}$ with the ATLAS detector, Phys. Rev. D 90 (2014) 052008 [arXiv:1407.0608] [INSPIRE].

[91] ATLAS missing energy trigger public results webpage, https://twiki.cern.ch/twiki/bin/view/AtlasPublic/MissingEtTriggerPublicResults.

[92] MadAnalysis 5 Public Analysis Database (PAD) for recasting LHC results webpage, http://madanalysis.irmp.ucl.ac.be/wiki/PublicAnalysisDatabase.

[93] J. Alwall, M. Herquet, F. Maltoni, O. Mattelaer and T. Stelzer, MadGraph 5: going beyond, JHEP 06 (2011) 128 [arXiv:1106.0522] [INSPIRE].

[94] J. Alwall et al., The automated computation of tree-level and next-to-leading order differential cross sections and their matching to parton shower simulations, JHEP $\mathbf{0 7}$ (2014) 079 [arXiv: 1405.0301] [INSPIRE].

[95] J. Pumplin, D.R. Stump, J. Huston, H.L. Lai, P.M. Nadolsky and W.K. Tung, New generation of parton distributions with uncertainties from global QCD analysis, JHEP $\mathbf{0 7}$ (2002) 012 [hep-ph/0201195] [INSPIRE].

[96] ATLAS collaboration, ATLAS tunes of PYTHIA 6 and PYTHIA 8 for MC11, ATL-PHYS-PUB-2011-009, CERN, Geneva Switzerland (2011). 
[97] ATLAS collaboration, Further ATLAS tunes of PYTHIA 6 and PYTHIA 8, ATL-PHYS-PUB-2011-014, CERN, Geneva Switzerland (2011).

[98] S. Frixione and B.R. Webber, Matching NLO QCD computations and parton shower simulations, JHEP 06 (2002) 029 [hep-ph/0204244] [INSPIRE].

[99] M.L. Mangano, M. Moretti, F. Piccinini and M. Treccani, Matching matrix elements and shower evolution for top-quark production in hadronic collisions, JHEP 01 (2007) 013 [hep-ph/0611129] [INSPIRE].

[100] T. Sjöstrand, S. Mrenna and P.Z. Skands, PYTHIA 6.4 physics and manual, JHEP 05 (2006) 026 [hep-ph/0603175] [INSPIRE].

[101] DELPHES 3 collaboration, J. de Favereau et al., DELPHES 3, a modular framework for fast simulation of a generic collider experiment, JHEP 02 (2014) 057 [arXiv:1307.6346] [INSPIRE].

[102] M. Krämer et al., Supersymmetry production cross sections in pp collisions at $\sqrt{s}=7 \mathrm{TeV}$, arXiv:1206.2892 [INSPIRE].

[103] LHC SUSY cross section working group webpage, https://twiki.cern.ch/twiki/bin/view/LHCPhysics/SUSYCrossSections.

[104] P.J. Fox, R. Harnik, J. Kopp and Y. Tsai, LEP shines light on dark matter, Phys. Rev. D 84 (2011) 014028 [arXiv: 1103.0240] [InSPIRE].

[105] P.J. Fox, R. Harnik, J. Kopp and Y. Tsai, Missing energy signatures of dark matter at the LHC, Phys. Rev. D 85 (2012) 056011 [arXiv:1109.4398] [INSPIRE].

[106] M. Cacciari, G.P. Salam and G. Soyez, FastJet user manual, Eur. Phys. J. C 72 (2012) 1896 [arXiv:1111.6097] [INSPIRE].

[107] M. Cacciari, G.P. Salam and G. Soyez, The anti-kt jet clustering algorithm, JHEP 04 (2008) 063 [arXiv: 0802.1189] [INSPIRE].

[108] C. Borschensky et al., Squark and gluino production cross sections in pp collisions at $\sqrt{s}=13,14,33$ and 100 TeV, Eur. Phys. J. C 74 (2014) 3174 [arXiv:1407.5066] [INSPIRE].

[109] A. Barr, C. Lester and P. Stephens, $m_{T 2}$ : the truth behind the glamour, J. Phys. G 29 (2003) 2343 [hep-ph/0304226] [INSPIRE].

[110] A.J. Barr and C. Gwenlan, The race for supersymmetry: using $m_{T 2}$ for discovery, Phys. Rev. D 80 (2009) 074007 [arXiv: 0907.2713] [InSPIRE].

[111] C.G. Lester and D.J. Summers, Measuring masses of semiinvisibly decaying particles pair produced at hadron colliders, Phys. Lett. B 463 (1999) 99 [hep-ph/9906349] [INSPIRE].

[112] G. Bélanger, D. Ghosh, R. Godbole, M. Guchait and D. Sengupta, Probing the flavor violating scalar top quark signal at the LHC, Phys. Rev. D 89 (2014) 015003 [arXiv: 1308.6484] [INSPIRE].

[113] Mono twiki webpage, https://atlas.web.cern.ch/Atlas/GROUPS/PHYSICS/PAPERS/SUSY-2013-21/. 